\title{
PIERRE PANSU
}

\section{Formules de Matsushima, de Garland et propriété (T) pour des groupes agissant sur des espaces symétriques ou des immeubles}

Bulletin de la S. M. F., tome 126, no 1 (1998), p. 107-139

<http://www.numdam.org/item?id=BSMF_1998_126_1_107_0>

(C) Bulletin de la S. M. F., 1998, tous droits réservés.

L'accès aux archives de la revue "Bulletin de la S. M. F. » (http: //smf.emath.fr/Publications/Bulletin/Presentation.html) implique l'accord avec les conditions générales d'utilisation (http://www.numdam.org/ conditions). Toute utilisation commerciale ou impression systématique est constitutive d'une infraction pénale. Toute copie ou impression de ce fichier doit contenir la présente mention de copyright.

\section{Numdam}




\title{
FORMULES DE MATSUSHIMA, DE GARLAND ET PROPRIÉTÉ (T) POUR DES GROUPES AGISSANT SUR DES ESPACES SYMÉTRIQUES OU DES IMMEUBLES
}

\author{
PAR PIERRE PANSU $(*)$
}

\begin{abstract}
RÉSUMÉ. - On explique l'origine géométrique de deux théorèmes d'annulation, l'un dû à Y. Matsushima concerne les variétés riemanniennes localement symétriques, l'autre, dû à H. Garland, concerne les immeubles. On montre que ces formules entraînent la propriété (T) de Kazhdan.

Abstract. - Matsushima's And Garland'S formulas. A geometric explanation of two classical vanishing theorems is given. These are Y. Matsushima's formula (concerning locally symmetric spaces) and its analogue for buildings discovered by H. Garland. Both formulae are shown to imply Kazhdan's property (T).
\end{abstract}

\section{Introduction}

En 1962, en s'inspirant de travaux d'André Weil, Y.Matsushima a découvert une variante de la formule de Bochner, spécifique aux variétés riemanniennes compactes localement symétriques $M$. Dans le cas où le rang de $M$ (i.e., la dimension maximale d'une sous-variété plate totalement géodésique) est au moins égal à 2 , cette formule implique que le premier nombre de Betti de $M$ est nul. Elle s'applique sans changement à la cohomologie à valeurs dans un système local unitaire de coefficients. Depuis, cette formule a été étendue aux formes différentielles de degré supérieur.

En 1972, H. Garland a découvert un analogue combinatoire de la formule de Matsushima, qui permet d'étendre le théorème d'annulation

(*) Texte reçu le 16 janvier 1996, révisé le 7 août 1997, accepté le 21 janvier 1998.

P. PANSu, URA D 1169 du CNRS, Mathématiques, Bât. 425, Université Paris-Sud, 91405 Orsay (France). Email : pierre.pansu@math.u-psud.fr.

Mots clés : théorème d'annulation, espace symétrique, immeuble, groupe de Kazhdan. Classification AMS : 22 D 10, 22 E40, 51E24. 
aux réseaux des groupes algébriques $p$-adiques (avec certaines restrictions sur $p$ qui ont été levées dans [BW]).

Dans cette note, on montre comment déduire les deux formules de l'invariance de la mesure de Liouville sur fibré des 2-plats par l'action naturelle de $\mathbb{R}^{2}$ (resp. $\mathbb{Z}^{2}$ ) sur cet espace. Ce point de vue a été inspiré par l'article [MSY] où N. Mok, Y.T. Siu et S.K. Yeung donnent une preuve nouvelle de certains résultats de superrigidité dûs à G.A. Margulis.

Les formules de Matsushima et de Garland permettent de démontrer rapidement que les groupes discrets cocompacts d'isométries de certains espaces symétriques et de certains immeubles euclidiens possèdent la propriété $(\mathrm{T})$ de Kazhdan, $[\mathrm{P}],[\mathrm{Mo}],[\mathrm{KS}],[\mathrm{Z}]$. En effet, la propriété $(\mathrm{T})$ équivaut à l'annulation de $H^{1}(\Gamma, \rho)$ pour toute représentation unitaire $\rho$ de $\Gamma$. Les formules entraînent de plus que, sous les mêmes hypothèse, $H^{2}(\Gamma, \rho)=0$ est séparé, (corollaires 6 et 20 ).

Même en l'absence de groupe d'isométrie (ce qui est le cas pour la plupart des immeubles triangulaires par exemple, [R], [RT], [T2], [S]), on peut tirer une conséquence géométrique des formules : l'annulation de l'espace $L^{2} H^{1}(X)$ de cohomologie $L^{2}$ et le fait que l'espace $L^{2} H^{2}(X)$ est séparé.

Enfin, notre approche de la formule de Garland conduit à une formule nouvelle pour les immeubles carrés, i.e., les produits d'arbres. Un groupe discret cocompact $\Gamma$ d'automorphismes d'un tel immeuble n'a jamais la propriété $(\mathrm{T})$, voir $[\mathrm{BS}],[\mathrm{NR}]$. Toutefois, si les projections de $\Gamma$ sur les facteurs sont suffisamment transitives, tout quotient de $\Gamma$ possède la propriété (T). C'est une étape dans la preuve par M. Burger et S. Mozes du fait qu'un tel quotient est nécessairement fini. Nous en donnons une preuve géométrique au paragraphe 10 .

Je remercie chaleureusement Étienne Ghys, qui a attiré mon attention sur les travaux de H. Garland, Alain Valette, dont la lecture critique de [P] est à l'origine de cette note, Jacques Tits et Frédéric Haglund pour les références sur les immeubles. Je dois beaucoup à Andrzej Zuk : c'est lui qui m'a expliqué que la version discrète de la formule de Matsushima coïncide avec la formule de Garland. Je remercie M. Burger et S. Mozes pour leurs explications sur les groupes agissant sur des produits d'arbres.

\section{Formules de Matsushima}

Voici comment N. Mok, Y.T. Siu et S.K. Yeung énoncent la formule de Matsushima.

Soit $E$ un espace vectoriel euclidien de dimension $n$. Un tenseur de courbure sur $E$ est un tenseur de $E^{*} \otimes E^{*} \otimes E^{*} \otimes E$ qui possède les mêmes

TOME $126-1998-\mathrm{N}^{\circ} 1$ 
symétries que le tenseur de courbure d'une variété riemannienne. Un tel tenseur $Q$ détermine une forme quadratique $Q$ sur les 2-tenseurs.

Proposition 1 (formule de Matsushima). - Soit $M$ une variété riemannienne compacte. Soit $Q$ un champ de tenseurs de courbure parallèle

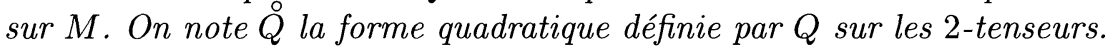
Soit $\alpha$ une 1-forme différentielle sur $M, D \alpha$ sa dérivée covariante. Alors

$$
\int_{M} Q(D \alpha)=-\frac{1}{2} \int_{M}\langle Q(\alpha), R(\alpha)\rangle .
$$

En particulier, si $M$ est localement symétrique et localement irréductible, alors

$$
\int_{M} Q(D \alpha)=-\frac{1}{2}\langle Q, R\rangle \int_{M}|\alpha|^{2} .
$$

Il y a un tenseur de courbure canonique, noté $I$, sur tout espace vectoriel euclidien, dont la courbure sectionnelle est constante et vaut 1 . La forme quadratique $\stackrel{\circ}{I}$ qui lui correspond est la suivante : si

$$
T=A+S+(\operatorname{tr} T) \frac{g}{n}
$$

est la décomposition du 2-tenseur $T$ en partie antisymétrique $A$, partie symétrique à trace nulle $S$ et composante colinéaire au produit scalaire, alors

$$
\stackrel{\circ}{I}(T)=|T|^{2}-|A|^{2}-(\operatorname{tr} T)^{2} .
$$

Toute variété riemannienne porte au moins un champ de tenseurs de courbure parallèle, le champ de tenseurs canonique $I$. Si on choisit $Q=I$, on obtient la formule de Bochner

$$
\int_{M}|D \alpha|^{2}-|\mathrm{d} \alpha|^{2}-(\delta \alpha)^{2}=-\int_{M} \operatorname{Ric}(\alpha)
$$

où $\delta$ est la codifférentielle et Ric la courbure de Ricci.

Sur une variété riemannienne, il y a autant de formules de Matsushima que de tenseurs de courbure invariants par holonomie. Noter que l'application $Q \mapsto{\stackrel{\circ}{\mid S^{2} T^{*}}}$ est injective. Par conséquent, la dimension de l'espace des tenseurs de courbure invariants par holonomie est au plus égale au nombre de composantes irréductibles $d(M)$ dans la décomposition sous l'holonomie de l'espace $S^{2} T^{*} M$.

Génériquement, $I$ est le seul choix possible, mais sur les variétés localement symétriques, le tenseur de courbure de Riemann $R$ lui-même 
est parallèle. Dans [M], Y. Matsushima prend pour $Q$ la projection $\mathrm{du}$ tenseur canonique $I$ sur l'hyperplan orthogonal au tenseur de courbure $R$.

Pour une variété localement symétrique, le nombre $d(M)$ de composantes est au plus égal à 4 , et vaut 2 le plus souvent (voir [WZ]).

\subsection{Généralisation.}

Pour les applications, il est utile de se placer sous des hypothèses plus générales. On supposera que $\Gamma$ est un groupe discret d'isométries d'une variété riemannienne $X$ (éventuellement $\Gamma$ a des points fixes et $\Gamma \backslash X$ est non compact). On se donne une représentation unitaire $\rho$ de $\Gamma$, éventuellement de dimension infinie. On considère alors des formes différentielles $L^{2}$ tordues par $\rho$. Une forme tordue par la représentation $\rho$ sur $\Gamma \backslash X$ est une forme $\alpha$ sur $X$ à valeurs dans l'espace de Hilbert $\mathcal{H}_{\rho}$ telle que, pour $\gamma \in \Gamma$, $\gamma^{*} \alpha=\rho(\gamma) \alpha$. La norme $|\alpha|^{2}$ devient une fonction sur $X$ invariante par $\Gamma$, dont l'intégrale sur $\Gamma \backslash X$ est appelée norme $L^{2}$ de $\alpha$. On dit que $D \alpha \in L^{2}$ si les dérivées partielles de $\alpha$ au sens des distributions sont localement des fonctions $L^{2}$, et si $|D \alpha| \in L^{2}(\Gamma \backslash X)$.

La proposition suivante se démontre comme dans [MSY].

Proposition 2. - Soient $X$ une variété riemannienne, $\Gamma$ un groupe discret d'isométries de $X, \rho$ une représentation unitaire de $\Gamma$. Soit $Q$ un champ de tenseurs de courbure parallèle sur $X$. Soit $\alpha$ une 1-forme différentielle sur $\Gamma \backslash X$ tordue par $\rho, D \alpha$ sa dérivée covariante. On suppose que $\alpha$ et $D \alpha \in L^{2}(\Gamma \backslash X)$. Alors

$$
\int_{\Gamma \backslash X} Q(D \alpha)+\frac{1}{2}\langle Q(\alpha), R(\alpha)\rangle=0 .
$$

Au paragraphe suivant, nous allons donner une nouvelle preuve de cette formule, pour des choix particuliers de $Q$.

\section{Formule de Matsushima et espace des plats}

On va faire apparaître une formule de Matsushima comme une moyenne de la formule de Bochner sur les sous-espaces plats d'un espace localement symétrique.

\subsection{La formule de Bochner sur un tore plat.}

Dans le tore plat riemannien $T=S^{1} \times S^{1}$, la formule de Bochner est simplement une double intégration par parties. Soit $\alpha=\alpha_{1} \mathrm{~d} x_{1}+\alpha_{2} \mathrm{~d} x_{2}$ une 1-forme. Alors $D \alpha$ est le 2-tenseur

$$
D \alpha=\sum_{i, j=1}^{2} \alpha_{i, j} \mathrm{~d} x_{i} \otimes \mathrm{d} x_{j}
$$

TOME $126-1998-\mathrm{N}^{\circ} 1$ 
où $\alpha_{i, j}=\partial \alpha_{i} / \partial x_{j}$. Aussi, $\delta \alpha=\alpha_{1,1}+\alpha_{2,2}, \mathrm{~d} \alpha=\left(\alpha_{1,2}-\alpha_{2,1}\right) \mathrm{d} x_{1} \wedge \mathrm{d} x_{2}$ d'où

$$
|D \alpha|^{2}=(\delta \alpha)^{2}+|\mathrm{d} \alpha|^{2}+2 Q(\alpha)
$$

où $Q(\alpha)=\alpha_{1,2} \alpha_{2,1}-\alpha_{1,1} \alpha_{2,2}$.

La formule de Bochner s'écrit

$$
\int_{T} Q(\alpha)=0
$$

et se démontre comme suit

$$
\int_{T} \alpha_{1,1} \alpha_{2,2}=-\int_{T} \alpha_{1,12} \alpha_{2}=-\int_{T} \alpha_{1,21} \alpha_{2}=\int_{T} \alpha_{1,2} \alpha_{2,1} .
$$

Autrement dit, on a écrit la fonction $Q(\alpha)$ comme combinaison de dérivées partielles de fonctions périodiques, $Q(\alpha)=v_{1}-u_{2}$ où

$$
u=\alpha_{2} \alpha_{1,1} \quad \text { et } \quad v=\alpha_{2} \alpha_{1,2},
$$

et utilisé le fait que les champs de vecteurs $\partial / \partial x_{i}$ engendrent sur le tore quotient une action de $\mathbb{R}^{2}$ qui préserve le volume.

\subsection{Nouvelle preuve d'une formule de Matsushima.}

Une lecture attentive de [MSY] suggère qu'on peut trouver une formule de Matsushima pour une variété riemannienne localement symétrique compacte $M=\Gamma \backslash G / K$ en choisissant un 2-plat marqué $f$ de $X=G / K$, en écrivant la formule de Bochner sur $f$ et sur ses translatés parallèles, et en faisant la moyenne.

Décrivons d'abord l'espace des plats marqués de $X=G / K$ en termes algébriques. Soit $G=N A K$ une décomposition d'Iwasawa. Dans le groupe abélien $A$ de $\operatorname{rang} r=\operatorname{rang}_{\mathbb{R}}(G)$, choisissons un sous groupe $A^{\prime}$ isomorphe à $\mathbb{R} \oplus \mathbb{R}$. Soit $K^{\prime}$ le centralisateur de $A^{\prime}$ dans $K$, et $\widetilde{F}=G / K^{\prime}$. Le groupe $A^{\prime}$ agit par translations à droite sur $\widetilde{F}$, en préservant la mesure de Haar $\mu$. La projection $\widetilde{F} \rightarrow G / K$ est une fibration, et la mesure $\mu$ se désintègre en la mesure riemannienne $\mathrm{d} x$ sur $X$ et une mesure $K$-invariante $\mu^{\prime}$ sur $K / K^{\prime}$.

Voici une construction plus géométrique de l'espace des plats marqués. Fixons un plan euclidien $\pi$, une origine $O \in \pi$ et un repère orthonormé $\left(e_{1}, e_{2}\right)$. Un plat marqué dans $M=\Gamma \backslash X$ est une immersion isométrique et totalement géodésique $f$ de $\pi$ dans $M$. On dit qu'un plat marqué $f^{\prime}$ est obtenu par transport parallèle à partir de $f$ s'il existe un chemin de $f(O)$ à $f^{\prime}(O)$ tel que le transport parallèle $\tau$ le long de ce chemin satisfasse $f_{*}^{\prime}=\tau \circ f_{*}$. 
Fixons un plat marqué $f_{0}$. Soit $F$ l'ensemble des plats marqués dans $M$ obtenus par transport parallèle à partir de $f_{0}$. Sur $F$ on a une action du groupe des translations de $\pi$, par précomposition. Cette action préserve bien $F$, car le transport parallèle le long des géodésiques d'un plat $f$ coïncide avec la précomposition par les translations. Alors $F$ (muni l'action des translations de $\pi$ ) n'est autre que le quotient de $\widetilde{F}$ par l'action à gauche de $\Gamma$.

À chaque plat marqué $f$ tel que $f(O)=x \in M$ correspond une forme quadratique $Q_{f}$, sur l'espace des formes bilinéaires $T$ sur $T_{x} M$, définie par

$$
Q_{f}(T)=T\left(f_{*} e_{1}, f_{*} e_{2}\right) T\left(f_{*} e_{2}, f_{*} e_{1}\right)-T\left(f_{*} e_{1}, f_{*} e_{1}\right) T\left(f_{*} e_{2}, f_{*} e_{2}\right) .
$$

À un champ $T$ de formes bilinéaires sur $M$ correspond donc une fonction $Q(T)$ sur l'espace $F$ des plats marqués. Cette construction s'étend aux formes bilinéaires à valeurs dans un espace de Hilbert $\mathcal{H}_{\rho}$ comme suit

$$
Q_{f}(T)=\left\langle T\left(f_{*} e_{1}, f_{*} e_{2}\right), T\left(f_{*} e_{2}, f_{*} e_{1}\right)\right\rangle-\left\langle T\left(f_{*} e_{1}, f_{*} e_{1}\right), T\left(f_{*} e_{2}, f_{*} e_{2}\right)\right\rangle .
$$

Soient $\alpha$ une 1-forme sur $M$ (éventuellement tordue), et $D \alpha$ sa dérivée covariante. Supposons que $\alpha$ et $D \alpha$ sont $L^{2}$. Pour chaque plat $f$ marqué en $x \in M$, on pose

$$
\begin{aligned}
& u_{f}(\alpha)=\left\langle\alpha\left(f_{*} e_{2}\right), D \alpha\left(f_{*} e_{1}, f_{*} e_{1}\right)\right\rangle, \\
& v_{f}(\alpha)=\left\langle\alpha\left(f_{*} e_{2}\right), D \alpha\left(f_{*} e_{1}, f_{*} e_{2}\right)\right\rangle .
\end{aligned}
$$

Alors $u(\alpha)$ et $v(\alpha)$ sont des fonctions $L^{1}$ sur l'espace $F$ des plats. Notant $\tau_{1}$ et $\tau_{2}$ les champs de vecteurs sur $F$ qui engendrent l'action naturelle de $\mathbb{R}^{2}$, on a, au sens des distributions,

$$
Q(D \alpha)=\tau_{1} v(\alpha)-\tau_{2} u(\alpha)
$$

ce qui entraîne

$$
\int_{F} Q_{f}(D \alpha) \mathrm{d} \mu(f)=0
$$

Posons, pour $x \in M, T$ une forme bilinéaire sur $T_{x} M$,

$$
Q_{x}(T)=\int_{\{f \mid f(O)=x\}} Q_{f}(T) \mathrm{d} \mu^{\prime}(f) .
$$

On obtient, pour toute 1-forme différentielle $\alpha$ sur $M$,

$$
\int_{M} Q_{x}(D \alpha) \mathrm{d} x=0
$$

TOME $126-1998-\mathrm{N}^{\circ} 1$ 
Ceci est bien une formule de Matsushima. En effet, pour chaque point $\tilde{x} \mathrm{du}$ revêtement universel $\widetilde{M}, Q_{\tilde{x}}$ est invariant sous le groupe d'isotropie $K$, qui coïncide avec le groupe d'holonomie, donc $Q$ est parallèle. D'autre part, comme le tenseur de courbure restreint à un plat $f$ est nul, chaque $Q_{f}$ est orthogonale au tenseur de courbure, et il en est de même de $Q_{\tilde{x}}$. Enfin, le produit scalaire de $Q_{f}$ avec le tenseur canonique $I$ vaut $\left\langle Q_{f}, I\right\rangle=4$ donc $\left\langle Q_{\tilde{x}}, I\right\rangle \neq 0$. En particulier, $Q$ n'est pas nul.

Question. - En faisant varier le 2-plat de référence, combien de tenseurs $Q$ différents obtient-on? Obtient-on toujours au moins un tenseur qui satisfait aux conclusions de la proposition 3 ? D'après [MSY], les seules exceptions potentielles sont les duaux non compacts des grassmanniennes.

\section{Cas des espaces localement symétriques de rang $\mathbf{1}$}

\subsection{La formule de Hodge pour le plan hyperbolique complexe.}

Soit $M$ une variété kählérienne, $\alpha$ une 1-forme sur $M$. On définit un opérateur différentiel du premier ordre $P$ par

$$
P \alpha(u, v)=D_{u} \alpha(v)+D_{J u} \alpha(J v) \text {. }
$$

Si de plus $M$ est compacte, alors on a la formule de Hodge

$$
\int_{M}|P \alpha|^{2}-|\mathrm{d} \alpha|^{2}-|\delta \alpha|^{2}=0
$$

Cette formule entraîne que toute 1-forme harmonique réelle sur $M$ est la partie réelle d'une 1-forme holomorphe.

Si $M$ est un quotient compact du plan hyperbolique complexe, la formule de Hodge coïncide avec la formule de Matsushima classique, i.e. celle obtenue lorsque $Q$ est la projection de $I$ sur l'orthogonal du tenseur de courbure.

\subsection{Formule de Corlette-Matsushima et espace des plans hyperboliques complexes.}

Soit $X$ un espace symétrique de rang 1 à courbure sectionnelle non constante. Alors il existe des plongements isométriques totalement géodésiques du plan hyperbolique complexe pointé $\left(\mathbb{C} H^{2}, x_{0}\right)$ dans $X$. L'ensemble de ces plongements est un fibré $P \rightarrow X$ homogène sous le groupe des isométries de $X$. Il possède donc une mesure invariante $\mu$. Le groupe Isom $\left(\mathbb{C} H^{2}\right)$ agit sur $P$ par précomposition. Ses orbites sont des copies de $\mathbb{C} H^{2}$ qui feuillettent $P$. 
Soit $\alpha$ une 1-forme sur $X$. Relevons $\alpha$ dans $P$. En chaque point $p \in P$ passe une feuille $\mathcal{F}(p)$. Notons

$$
Q_{p}(\alpha)=\left|P\left(\alpha_{\mid \mathcal{F}(p)}\right)\right|^{2}-\left|\mathrm{d}\left(\alpha_{\mid \mathcal{F}(p)}\right)\right|^{2}-\left|\delta\left(\alpha_{\mid \mathcal{F}(p)}\right)\right|^{2}
$$

évalué au point $p$. En intégrant sur la fibre, on obtient une expression quadratique par rapport aux dérivées premières de $\alpha$, notée $Q_{x}(D \alpha)$.

La forme quadratique $Q_{x}$ sur l'espace des 2-tenseurs de l'espace tangent $T X$ est invariante par le stabilisateur $K$ de $x$. Elle est donc parallèle. Les produits scalaires $\left\langle Q_{p}, R\right\rangle$ et $\left\langle Q_{p}, I\right\rangle$ sont des constantes universelles, qu'on évalue sur un quotient compact de $\mathbb{C} H^{2}$. On trouve, en comparant les formules de Hodge et de Matsushima, que $\left\langle Q_{p}, R\right\rangle=0$ et $\left\langle Q_{p}, I\right\rangle \neq 0$. Par conséquent $\left\langle Q_{x}, R\right\rangle=0$ mais $Q_{p} \neq 0$. Comme le nombre $d(M)$ de composantes irréductibles dans la décomposition sous $K$ de l'espace des 2-tenseurs symétriques sur l'espace tangent vaut 2 pour les espaces considérés, on conclut que $Q_{x}$ est un multiple non nul de la projection de $I$ sur l'orthogonal de $R$. Autrement dit, la formule de Matsushima n'est autre que

$$
\int_{\Gamma \backslash P} Q_{p}(\alpha) \mathrm{d} p=0,
$$

c'est-à-dire la formule de Hodge moyennée sur les plans hyperboliques complexes de $\Gamma \backslash X$.

Pour l'espace hyperbolique quaternionnien ou le plan hyperbolique sur les octaves de Cayley, la formule de Matsushima a été trouvée par K. Corlette [C].

\section{Applications de la formule de Matsushima}

On donne les détails de l'argument évoqué dans [P, p. 89]. Les applications découlent de la propriété de positivité suivante.

Proposition 3. - Soit $X$ un espace symétrique irréductible distinct de l'espace hyperbolique réel ou complexe. Il existe sur $X$ un tenseur de courbure parallèle tel que $\langle Q, R\rangle=0$ et tel que la forme quadratique $\stackrel{\odot}{Q}$, restreinte aux formes bilinéaires symétriques à trace nulle, soit définie positive.

La preuve de cette proposition repose en bonne part sur un examen cas par cas de la classification des espaces symétriques. Elle est due à de nombreux auteurs, depuis E. Calabi, E. Vesentini et A. Borel en 1960 jusqu'à N. Mok, Y.T. Siu et S.K. Yeung en 1993, voir [MSY].

On tire d'abord de la formule une inégalité.

TOME $126-1998-\mathrm{N}^{\circ} 1$ 
Lemme 4. - Soit $X$ un espace symétrique irréductible distinct de l'espace hyperbolique réel ou complexe, $\Gamma$ un groupe discret d'isométries de $X, \rho$ une représentation unitaire de $\Gamma$. Soit $\alpha$ une 1-forme différentielle sur $\Gamma \backslash X$ tordue par $\rho, D \alpha$ sa dérivée covariante. On suppose que $\alpha, \mathrm{d} \alpha$ et $\delta \alpha$ sont dans $L^{2}(\Gamma \backslash X)$. Alors il existe une constante $C$ indépendante de la représentation $\rho$ telle que

$$
\|\alpha\|^{2}+\|D \alpha\|^{2} \leq C\left(\|\delta \alpha\|^{2}+\|\mathrm{d} \alpha\|^{2}\right)
$$

Preuve. - Soit $X$ un espace symétrique irréductible de rang $>1$. Soit $Q$ un champ de tenseurs de courbure parallèle sur $X$ tel que $\langle Q, R\rangle=0$. La forme quadratique $\langle Q(\xi), R(\xi)\rangle$ sur l'espace cotangent est $K$-invariante, donc proportionnelle à la métrique. $\mathrm{Si}\langle Q, R\rangle=0$, elle est identiquement nulle, donc $\langle Q(\xi), R(\xi)\rangle=0$ pour tout $\xi \in T^{*} X$.

D'après 3 , on peut choisir un champ de tenseurs de courbure parallèle $Q$ tel que $\langle Q, R\rangle=0$ et que la forme quadratique $\stackrel{\circ}{Q}$ restreinte aux formes bilinéaires symétriques à trace nulle soit définie positive. Il existe alors des constantes $C^{\prime}$ et $C^{\prime \prime}$ telles que, pour toute forme bilinéaire $T$ sur $T X$,

$$
\langle T, T\rangle \leq C^{\prime}\left(|\mathcal{A}(T)|^{2}+|\operatorname{tr} T|^{2}\right)+C^{\prime \prime} \stackrel{\circ}{Q}(T)
$$

où $\mathcal{A}(T)(u, v)=T(u, v)-T(v, u)$. La même inégalité, avec les mêmes constantes, est vraie pour des formes bilinéaires à valeurs dans un espace de Hilbert $\mathcal{H}_{\rho}$.

Soit $\alpha$ une 1-forme différentielle sur $\Gamma \backslash X$ tordue par $\rho$. Comme $\mathrm{d} \alpha=\mathcal{A}(D \alpha)$ et $\delta \alpha=\operatorname{tr} D \alpha$, il vient

$$
|D \alpha|^{2} \leq C^{\prime}\left(|\mathrm{d} \alpha|^{2}+|\delta \alpha|^{2}\right)+C^{\prime \prime} \stackrel{\circ}{Q}(D \alpha) .
$$

Lorsque $\alpha$ et $D \alpha$ sont dans $L^{2}$, la formule de Matsushima s'écrit $\int \stackrel{\circ}{Q}(D \alpha)=0$, d'où en intégrant l'inégalité $\left(^{*}\right)$,

$$
\|D \alpha\|^{2} \leq C^{\prime}\left(\|\mathrm{d} \alpha\|^{2}+\|\delta \alpha\|^{2}\right)
$$

Toujours sous l'hypothèse $\alpha, D \alpha \in L^{2}$, la formule de Bochner donne

$$
\|D \alpha\|^{2}-\|\delta \alpha\|^{2}-\|\mathrm{d} \alpha\|^{2}=(-\mathrm{Ric})\|\alpha\|^{2}
$$

où -Ric est une constante strictement positive. Il existe donc une constante $C$ telle que

$$
\|\alpha\|_{L^{1,2}}^{2}:=\|\alpha\|^{2}+\|D \alpha\|^{2} \leq C\left(\|\mathrm{~d} \alpha\|^{2}+\|\delta \alpha\|^{2}\right) .
$$


Il reste à montrer que $\alpha, \mathrm{d} \alpha, \delta \alpha \in L^{2}$ entraîne $D \alpha \in L^{2}$. En vertu de l'inégalité $\left({ }^{* \prime}\right)$, il suffit de montrer que $\alpha$ est limite au sens des distributions de formes $\alpha_{j}$ telles que $\left\|\mathrm{d} \alpha_{j}\right\|$ et $\left\|\delta \alpha_{j}\right\|$ restent bornés. Comme l'orbifold $\Gamma \backslash X$ est complète, en multipliant $\alpha$ par des fonctions de la distance à un point, on approche $\alpha$ par des formes à support compact. On peut alors supposer que le support de $\alpha$ est arbitrairement petit, i.e. que $\alpha$ est à valeurs dans un espace de Hilbert fixe $\mathcal{H}_{\rho}$. Fixant une base hilbertienne de $\mathcal{H}_{\rho}$, on approche chaque composante de $\alpha$ par des formes différentielles scalaires à coefficients de classe $C^{1}$. Cela donne la densité cherchée.

Corollaire 5. - Sous les mêmes hypothèses, on obtient des conséquences sur la cohomologie $L^{2}$ de $\Gamma \backslash X$ tordue par $\rho$ :

- l'espace $L^{2} H^{1}(\Gamma \backslash X, \rho)$ est nul et

- l'espace $L^{2} H^{2}(\Gamma \backslash X, \rho)$ est séparé.

Preuve. - Il convient de préciser ce qu'on entend par cohomologie $L^{2}$.

Soit $M$ une variété riemannienne. Soit $L^{2} \Omega^{k}(M)$ l'espace des $k$-formes $\alpha \in L^{2}(M)$ dont la différentielle extérieure $\mathrm{d} \alpha$, en tant que distribution, coïncide avec une $k+1$-forme $L^{2}$. Muni de la norme $\|\alpha\|_{L^{2}}+\|\mathrm{d} \alpha\|_{L^{2}}$, $L^{2} \Omega^{k}(M)$ est un espace de Hilbert et d : $L^{2} \Omega^{k}(M) \rightarrow L^{2} \Omega^{k+1}(M)$ est un opérateur borné. Par définition, la cohomologie $L^{2}$ de $M$ est la cohomologie du complexe $\left(L^{2} \Omega^{*}(M), \mathrm{d}\right)$.

Notons :

- $\mathcal{H}_{0}$ l'espace des 1 -formes fermées $L^{2}$ tordues par $\rho$;

- $\mathcal{H}_{1}$ l'espace des 1 -formes fermées $L^{2}$ tordues par $\rho$ dont la dérivée covariante est $L^{2}$, muni de la norme $L^{1,2}$;

- $\mathcal{J}$ l'espace des 0 -formes $L^{2}$ tordues par $\rho$.

De l'inégalité ( $\left(\right.$ )), on tire d'abord que l'opérateur $\delta: \mathcal{H}_{1} \rightarrow \mathcal{J}$ a une image fermée, notée $\mathcal{I}$. Ensuite, que $\delta$ est un isomorphisme de $\mathcal{H}_{1}$ sur $\mathcal{I}$. Son inverse $\delta^{-1}$ est a fortiori borné de $\mathcal{I}$ dans $\mathcal{H}_{0}$, donc a un adjoint $L^{2}$ partout défini $\left(\delta^{-1}\right)^{*}: \mathcal{H}_{0} \rightarrow \mathcal{I}$.

Si $\alpha$ est une 1 -forme fermée $L^{2}$ tordue par $\rho$, i.e., $\alpha \in \mathcal{H}_{0}$, la 0 -forme $\beta=\left(\delta^{-1}\right)^{*} \alpha \in \mathcal{I}$ satisfait, pour tout $\beta^{\prime} \in \mathcal{H}_{1}$,

$$
\left\langle\delta \beta^{\prime}, \beta\right\rangle_{L^{2}}=\left\langle\beta^{\prime}, \alpha\right\rangle_{L^{2}}
$$

Par conséquent, au sens des distributions, $\mathrm{d} \beta=\alpha$, donc $\beta \in L^{2} \Omega^{0}$. On a donc montré l'annulation du premier espace de cohomologie $L^{2}$ de $\Gamma \backslash X$ tordue par la représentation $\rho$.

D'autre part, l'inégalité ( $($ ) entraîne que l'image de la différentielle extérieure d : $L^{2} \Omega^{1} \rightarrow L^{2} \Omega^{2}$ est fermée. En effet, soit $\alpha_{j} \in L^{2} \Omega^{1}$ des 1 -formes

TOME $126-1998-\mathrm{N}^{\circ} 1$ 
telles que $\beta_{j}=\mathrm{d} \alpha_{j}$ converge vers $\beta$ dans $L^{2} \Omega^{2}$. En choisissant $\alpha_{j}$ de norme minimum parmi les primitives de $\beta_{j}$ (c'est un sous-espace affine fermé), on peut supposer que $\delta \alpha_{j}=0$. L'inégalité ( $\downarrow$ ) donne une borne a priori sur la norme $L^{2}$ de $\alpha_{j}$. On peut donc supposer que $\alpha_{j}$ converge faiblement vers $\alpha \in L^{2} \Omega^{1}$. Alors $\mathrm{d} \alpha_{j}$ converge au sens des distributions vers $\mathrm{d} \alpha$, donc $\beta=\mathrm{d} \alpha$ est dans l'image de $\mathrm{d}$. On conclut que le second espace de cohomologie $L^{2}$ de $\Gamma \backslash X$ tordue par la représentation $\rho$ est séparé.

Corollaire 6. - Soient $X$ est un espace symétrique irréductible distinct de l'espace hyperbolique réel ou complexe, $\Gamma$ un groupe discret cocompact d'isométries de $X, \rho$ une représentation unitaire de $\Gamma$. Alors

$$
H^{1}(\Gamma, \rho)=0 \quad \text { et } \quad H^{2}(\Gamma, \rho) \quad \text { est séparé. }
$$

En particulier, $\Gamma$ possède la propriété $(\mathrm{T})$ de Kazhdan et est «big》 au sens de J. Lott, voir [Lo, section 5.1].

En effet, comme $X$ est contractile, $H^{*}(\Gamma, \rho)=H^{*}(\Gamma \backslash X, \rho)$. C'est clair lorsque $\Gamma$ agit librement, et cela reste vrai en général car les groupes d'isotropie sont finis et les coefficients sont pris dans un espace vectoriel sur un corps. Si $\Gamma \backslash X$ est compact, la condition $L^{2}$ ne change pas la cohomologie, et $H^{1}(\Gamma, \rho)=L^{2} H^{1}(\Gamma \backslash X, \rho)$.

D'après A. Guichardet, [Gu, p. 288], la propriété (T) pour un groupe $\Gamma$ résulte de l'annulation de $H^{1}(\Gamma, \rho)$ pour toute représentation unitaire $\rho$, et lui est en fait équivalente, [DV, p. 48]. Un groupe infini discret qui a la propriété $(\mathrm{T})$ est non moyennable

Sur les 1-formes cofermées, l'inégalité $\left({ }^{* \prime}\right)$ s'écrit

$$
\|\alpha\|^{2} \leq C\langle\alpha, \Delta \alpha\rangle
$$

Autrement dit, $\Delta$ est inversible sur les 1-formes cofermées tordues par $\rho$. En particulier, Ker $\Delta=0$ et $\Delta$ est inversible sur $L^{2} \Omega^{1} \bmod \operatorname{Ker} d$. Lorsque $\rho$ est la représentation régulière $\ell^{2}(\Gamma)$, ce résultat se traduit en termes de cohomologie $L^{2}$ du groupe $\Gamma$, et exprime qu'il est «big 》 au sens de J. Lott.

Remarque 7. - Un groupe est «big》 au sens de J. Lott si et seulement si, pour un (et donc tout) complexe qui calcule $L^{2} H^{*}\left(\Gamma, \ell^{2}(\Gamma)\right)$, la différentielle en degrés 0 et 1 admet un inverse borné.

Remarque 8. - Le corollaire 6 s'étend à certains sous-groupes discrets $\Gamma$ de covolume fini, $c f$. [GR]. 
Voici une condition suffisante qui ne dépend que de l'espace symétrique $X$. Soit $M=\Gamma \backslash X$. D'après [BoS], il existe une rétraction $\pi$ de $M$ sur une sous-variété à coins compacte $N$ de $M$. Si on peut choisir $\pi$ de sorte que $\mathrm{d} \pi \in L^{2}(M)$, alors toute classe $c \in H^{1}(M, \rho)$ est représentée par une 1-forme $L^{2}$. En effet, $c$ provient de $N$, donc est représentée par une 1-forme de la forme $\alpha=\pi^{*} \beta, \beta$ est bornée et $\alpha \in L^{2}$. La nullité de $H^{1}(\Gamma, \rho)=H^{1}(M, \rho)$ résulte alors de celle de $L^{2} H^{1}(M, \rho)$.

Le bord de $N$ est constitué de morceaux d'horosphères. À distance $r$, la norme de la différentielle de la projection orthogonale sur l'horosphère orthogonale à un vecteur $v$ d'une chambre de Weyl $W$ est le plus grand des nombres $\mathrm{e}^{r \lambda(X)}$ où $\lambda$ décrit les racines positives relatives à $W$. En revanche, à un terme polynômial près, le volume croît comme $\mathrm{e}^{-r \mu(X)}$ où $\mu$ est la demi-somme des racines positives. Un examen des racines conduit donc à une condition suffisante.

Cette condition suffisante est satisfaite pour tous les espaces symétriques de rang un à l'exception de $\mathbb{R} H^{2}, \mathbb{R} H^{3}$ et $\mathbf{C} H^{2}$. Elle est satisfaite aussi pour l'espace symétrique de $\operatorname{Sl}(n, \mathbb{R})$ dès que $n \geq 4$.

Remarque 9. - La méthode donne en principe, pour chaque système générateur fini $S$ de $\Gamma$, une estimation de la constante de Kazhdan indépendante de la représentation unitaire $\rho, c f$. $[\mathrm{Bu}],[\mathrm{KT}]$.

La constante de Kazhdan de $\rho$ pour le système générateur $S$ est le plus grand $\epsilon$ tel que, pour tout vecteur $\xi \in \mathcal{H}_{\rho}$,

$$
\max _{\gamma \in S}|\rho(\gamma) \xi-\xi| \geq \epsilon|\xi|
$$

Sur l'espace $Z^{1}(\Gamma, \rho)$ des 1-cocycles sur $\Gamma$ à valeurs dans $\rho$, utilisons la norme

$$
\|c\|^{2}=\sum_{\gamma \in S}|c(\gamma)|^{2}
$$

et sur l'espace $C^{0}(\Gamma, \rho)=\mathcal{H}_{\rho}$ la norme donnée. Supposons que $\rho$ n'a pas de vecteurs invariants non nuls. L'annulation de $H^{1}(\Gamma, \rho)$ signifie que

$$
\delta: C^{0}(\Gamma, \rho) \longrightarrow Z^{1}(\Gamma, \rho), \quad \xi \longmapsto c(\gamma)=\rho(\gamma) \xi-\xi
$$

est inversible, et estimer $\epsilon$, c'est estimer la norme de l'inverse $\delta^{-1}$. De telles bornes se transportent lorsqu'on remplace un complexe par un autre complexe qui lui est homotope (avec homotopies bornées). C'est le cas lorsqu'on passe de la résolution standard ci-dessus au complexe de de Rham sur $\Gamma \backslash X$, voir $[\mathrm{Br}]$. Les constantes dépendent de la géométrie de $\Gamma \backslash X$ de façon compliquée. 


\section{Formule de Garland dans le cas des immeubles triangulaires}

\subsection{Complexes simpliciaux.}

Soit $X$ un complexe simplicial. Une cochaîne simpliciale de degré $k$ sur $X$ est une fonction antisymétrique sur l'ensemble $\Sigma(k)$ des $k$-simplexes orientés de $X$. L'espace $C^{k}(X)$ des $k$-cochaînes est muni de la norme $\ell^{2}$

$$
\|\eta\|^{2}=\frac{1}{2} \sum_{\sigma \in \Sigma(k)}|\eta(\sigma)|^{2} .
$$

Si $\sigma$ est un $k$-simplexe orienté et $\tau$ un $(k-1)$-simplexe orienté, le coefficient d'incidence $[\sigma: \tau]$ vaut 1 (resp. -1 ) si $\tau$ est une face de $\sigma$ munie de l'orientation induite (resp. opposée) par $\sigma$, et 0 sinon. La différentielle $\mathrm{d}: C^{k}(X) \rightarrow C^{k+1}(X)$ est définie par

$$
\mathrm{d} \eta(\sigma)=\sum_{\tau \in \Sigma(k)}[\sigma: \tau] \eta(\tau) .
$$

Son adjoint $\delta$ pour la norme $\ell^{2}$ est donné par la formule

$$
\delta \eta(\sigma)=\sum_{\tau \in \Sigma(k+1)}[\tau: \sigma] \eta(\tau)
$$

La cohomologie (resp. $L^{2}$ ) du complexe des cochaînes simpliciales (resp. $L^{2}$ ) coïncide avec la cohomologie (resp. $L^{2}$ ) de l'espace topologique $X$. Plus généralement, étant donné un système local de coefficients (i.e. une représentation unitaire $\rho$ de $\pi_{1}(X)$ ), la cohomologie (resp. $L^{2}$ ) à coefficients dans $\rho$ est calculée par le complexe des cochaînes simpliciales tordues par $\rho$. Une cochaîne tordue par $\rho$ est une cochaîne sur le revêtement universel $\widetilde{X}$ à valeurs dans $\mathcal{H}_{\rho}$, équivariante sous $\pi_{1}(X)$. Plus généralement, si $\Gamma$ est un groupe discret d'automorphismes de $X$, on appelera cochaîne tordue par $\rho$ sur $\Gamma \backslash X$ une cochaîne $\Gamma$-équivariante sur $X$. La norme $L^{2}$ s'obtient en sommant sur $\Gamma \backslash \Sigma(k)$.

Soit $s$ un sommet de $X$. L'étoile de $s$ est la réunion des simplexes qui contiennent $s$. Le link de $X$ en $s$ est la réunion des simplexes de l'étoile qui ne contiennent pas $s$. Autrement dit, un $(k-1)$-simplexe $\sigma=\left(s_{1}, \cdots, s_{k}\right)$ est dans $\ell k(s)$ si et seulement si le cône $s(\sigma)=\left(s, s_{1}, \cdots, s_{k}\right)$ est un $k$ simplexe de $X$. Étant donné une cochaîne $\eta$ sur $X$ et un sommet $s$ de $X$, la formule

$$
\text { cône }_{s}(\eta)(\sigma)=\eta\left(\text { cône }_{s}(\sigma)\right)
$$

définit une cochaîne sur le link $\ell k(s)$. Cette opération ne commute pas avec la différentielle, si bien qu'on note $D \eta(s)$ le couple $\left(\right.$ cône $_{s}(\eta)$, cône $s(\mathrm{~d} \eta)$ ). 
Par exemple, si $X$ est de dimension 2 et $\eta$ est une 1-cochaîne,

$$
D \eta(s)=(u, v)
$$

où $u$ est la fonction sur l'ensemble des sommets du link $\ell k(s)$ définie par $u(y)=\eta(s, y)$ et $v$ la fonction sur l'ensemble des arêtes orientées du link définie par $v(y, z)=\mathrm{d} \eta(s, y, z)=\eta(s, y)+\eta(y, z)+\eta(z, s)$.

\subsection{Cas particulier des graphes.}

Si $L$ est un graphe fini, alors $C^{0}(L)$ est l'espace des fonctions sur l'ensemble des sommets et $C^{1}(L)$ l'espace des fonctions $v$ sur les couples de points voisins qui satisfont $v(y, z)=-v(z, y)$. Pour $u \in C^{0}(L)$,

$$
\mathrm{d} u(y, z)=u(z)-u(y) .
$$

Étant donné un sommet $y$ de $L$, on note $V(y)$ l'ensemble de ses voisins. Pour $v \in C^{1}(L)$,

$$
\delta v(y)=-\sum_{z \in V(y)} v(y, z) .
$$

\subsection{Immeubles triangulaires.}

Soit $\mathcal{P}$ un plan projectif fini. On note $q+1$ le nombre de droites passant par un point donné de $\mathcal{P}$. On appelle $q$ l'ordre du plan $\mathcal{P}$. Le graphe d'incidence de $\mathcal{P}$ a pour sommets les points et les droites de $\mathcal{P}$ (soit $2 q^{2}+2 q+2$ sommets) et une arête pour chaque paire $\{p, \ell\}$ où $p$ est un point de la droite $\ell$.

Un graphe fini $L$ est le graphe d'incidence d'un plan projectif si et seulement si :

- tout cycle de $L$ est de longueur au moins égale à 6 ;

- deux arêtes de $L$ sont contenues dans un même cycle de longueur 6 .

DÉFINITION 10. - Un immeuble triangulaire est un complexe simplicial simplement connexe de dimension deux dont chaque link est le graphe d'incidence d'un plan projectif fini. Tous les links ont même ordre, qu'on appelle l'ordre de l'immeuble.

Proposition 11 (formule de Garland). - Soit $X$ un immeuble triangulaire d'ordre $q$. Soit $\Gamma$ un groupe discret d'automorphismes de $X$. Soit $\rho$ une représentation unitaire de $\Gamma$. Soit $\eta$ une 1-cochaîne $L^{2}$ sur $\Gamma \backslash X$ tordue par $\rho$. Si $D \eta(s)=(u, v)$, on note, pour chaque sommet $s$ de $X$,

$$
\begin{aligned}
Q_{s}(D \eta) & =\langle\mathrm{d} u, \mathrm{~d} u\rangle-(q+1)\langle u, u\rangle \\
& -\frac{1}{3 q}\langle v, v\rangle+\frac{1}{12 q}\langle\delta v, \delta v\rangle+\frac{1}{6 q}\langle(2 q-4) u+\delta \mathrm{d} u, \delta v\rangle .
\end{aligned}
$$

TOME $126-1998-\mathrm{N}^{\circ} 1$ 
Alors

$$
\sum_{s \in \Gamma \backslash X} Q_{s}(D \eta)=0
$$

\section{Nouvelle preuve de la formule de Garland}

\subsection{Formule de Bochner simpliciale.}

Soit $\pi$ un complexe simplicial isométrique au plan euclidien, i.e., un plan euclidien pavé par des triangles équilatéraux. On choisit deux translations $\tau$ et $\tau^{\prime}$ par des vecteurs unitaires parallèles aux côtés des simplexes, et faisant entre eux un angle de $120^{\circ}$.

On va démontrer un analogue combinatoire de la formule de Bochner pour le quotient de $\pi$ par un réseau de translations simpliciales $\Gamma$.

Si $\eta$ est une 1-cochaîne de $\pi$, on définit une fonction $Q(\eta)$ sur les sommets $s$ de $\pi$ par la formule

$$
\begin{aligned}
Q_{s}(\eta)=( & \left.\eta\left(\tau^{\prime} s, \tau^{\prime} \tau s\right)-\eta(s, \tau s)\right)\left(\eta\left(\tau s, \tau \tau^{\prime} s\right)-\eta\left(s, \tau^{\prime} s\right)\right) \\
& -\left(\eta(s, \tau s)-\eta\left(\tau^{-1} s, s\right)\right)\left(\eta\left(s, \tau^{\prime} s\right)-\eta\left(\tau^{\prime-1} s, s\right)\right)
\end{aligned}
$$

Vérifions que, si $\eta$ est $\Gamma$-périodique, la somme des valeurs de $Q(\eta)$ sur les sommets d'une maille est nulle. Posons

$$
\begin{aligned}
& u(s)=\left(\eta(s, \tau s)-\eta\left(\tau^{-1} s, s\right)\right) \eta\left(s, \tau^{\prime} s\right), \\
& v(s)=\left(\eta\left(\tau^{\prime} s, \tau^{\prime} \tau s\right)-\eta(s, \tau s)\right) \eta\left(s, \tau^{\prime} s\right) .
\end{aligned}
$$

Par construction, $Q(\eta)=u-u \circ \tau^{\prime}-v+v \circ \tau$. Comme $\tau$ et $\tau^{\prime}$ commutent à $\Gamma$, elles définissent des bijections du quotient, donc

$$
\sum_{s \text { sommet de } \Gamma \backslash \pi} u \circ \tau^{\prime}-u=0
$$

et idem pour $v$.

REMARQUe 12. - L'expression $Q_{s}(\eta)$ ne dépend que de la restriction de $\eta$ à l'hexagone formé par les six voisins de $s$. Pour vérifier que sa somme est nulle (sur un quotient), on n'a eu besoin que de deux translations $\tau$ et $\tau^{\prime}$ qui passent au quotient et préservent la mesure de comptage.

\subsection{Vers la formule de Garland.}

Lorsque le tore est remplacé par un immeuble triangulaire $X$, l'expression $Q_{s}(\eta)$ est définie comme suit. A chaque plat $f$ marqué en $s$ correspond 
une expression $Q_{f}(\eta)$, on en fait la moyenne par rapport à une mesure $\mu_{s}$. Comme $Q_{f}(\eta)$ ne dépend que de la trace de $f$ dans l'étoile de $s, Q_{s}(\eta)$ ne dépend que de la restriction de $\eta$ à l'étoile de $s$.

L'invariance de la mesure $\mu=\mu_{s} \otimes \mathrm{d} s$ par l'action naturelle de $\mathbb{Z} \oplus \mathbb{Z}$ sur l'espace des plats marqués entraîne que la somme des valeurs de $Q(\eta)$ sur tout quotient est nulle.

Bien sûr, la preuve donnée par H. Garland est beaucoup plus courte, voir $[G]$.

\subsection{Plats marqués.}

On fixe un plan euclidien triangulé $\pi_{0}$. On fixe une origine $O$ et on note $y^{1}, \cdots, y^{6}$ les six sommets voisins de $O$ qui forment le link $\ell k_{\pi_{0}}(O)$, pris dans l'un des deux ordres circulaires possibles. On note $\tau_{0}$ la translation de vecteur unitaire $O y_{1}$ et $\tau_{0}^{\prime}$ la translation de vecteur unitaire $O y_{3}$.

DÉFINITION 13. - On appellera plat marqué un plongement isométrique $f: \pi_{0} \rightarrow X$. On note $F$ l'ensemble des plats marqués.

Sur $F$, on a une action de $\mathbb{Z} \oplus \mathbb{Z}$, i.e., deux bijections $\tau$ et $\tau^{\prime}$ définies comme suit. Si $f: \pi_{0} \rightarrow X$, alors $\tau(f)=f \circ \tau_{0}$ et $\tau^{\prime}(f)=f \circ \tau_{0}^{\prime}$.

Il faut voir cette action comme une sorte de flot géodésique.

On note $\mathcal{F}_{s}$ l'ensemble des plats marqués tels que $f(O)=s$.

Étant donné un 6-cycle marqué $\xi=\left(y_{1}, \cdots, y_{6}\right)$ du link $\ell k(s)$, on dit qu'un plat marqué $f: \pi_{0} \rightarrow X$ contient $\xi$ si $f(O)=s$ et $f\left(y^{i}\right)=y_{i}$. On note $\mathcal{F}_{\xi}$ l'ensemble des plats marqués en $s$ et contenant $\xi$.

Proposition 14. - Il existe une mesure $\mu$ sur $F$ ayant les propriétés suivantes.

(i) le nombre $\mu\left(\mathcal{F}_{\xi}\right)$ est indépendant du 6-cycle marqué $\xi$ (on le normalise de sorte que $\left.\mu\left(\mathcal{F}_{s}\right)=1\right)$;

(ii) $\mu$ est invariante par l'action naturelle de $\mathbb{Z} \oplus \mathbb{Z}$;

(iii) $\mu$ est invariante par tout automorphisme de l'immeuble $X$.

Noter qu'un polyèdre à courbure négative ou nulle possède toujours un flot géodésique. Dans [BB], W. Ballmann et $\mathrm{M}$. Brin ont construit une mesure de Liouville, invariante par ce flot. Ici, nous considérons un cas particulier, les polyèdres formés de triangles euclidiens, nous étudions essentiellement la même mesure et utilisons son invariance par une action qui n'existe pas dans le cas général. La preuve de la proposition 14 sera donnée au paragraphe 8 .

TOME $126-1998-\mathrm{N}^{\circ} 1$ 


\subsection{Intégration par parties.}

Soit $X$ un immeuble triangulaire. Soit $\Gamma$ un groupe discret d'automorphismes de $X$. Soit $\rho$ une représentation unitaire de $\Gamma$.

L'espace des plats marqués $F$ possède une structure de complexe simplicial (non localement fini) définie comme suit. Deux (resp. trois) points de $F$ sont les sommets d'un même simplexe de $F$ si et seulement si ils se trouvent dans une même orbite de $\mathbb{Z} \oplus \mathbb{Z}$ et leurs projections dans $X$ sont les sommets d'un simplexe de $X$.

Soit $\eta$ une 1-cochaîne $L^{2}$ sur $\Gamma \backslash X$ tordue par $\rho$. On la relève en une cochaîne $\tilde{\eta}$ sur l'espace des plats marqués $F$ comme suit : si $\widetilde{\chi}$ se projette sur $\chi$, alors $\tilde{\eta}(\tilde{\chi})=\eta(\chi)$.

On définit des fonctions $u$ et $v$ sur $F$ par les formules

$$
\begin{aligned}
& u(f)=\left\langle\tilde{\eta}(f, \tau f)-\tilde{\eta}\left(\tau^{-1} f, f\right), \tilde{\eta}\left(f, \tau^{\prime} f\right)\right\rangle, \\
& v(f)=\left\langle\tilde{\eta}\left(\tau^{\prime} f, \tau^{\prime} \tau f\right)-\tilde{\eta}(f, \tau f), \tilde{\eta}\left(f, \tau^{\prime} f\right)\right\rangle .
\end{aligned}
$$

et on pose

$$
\begin{aligned}
Q_{f}(\eta) & =\left(u-u \circ \tau^{\prime}-v+v \circ \tau\right)(f) \\
& =\left\langle\tilde{\eta}\left(\tau^{\prime} f, \tau^{\prime} \tau f\right)-\tilde{\eta}(f, \tau f), \tilde{\eta}\left(\tau f, \tau \tau^{\prime} f\right)-\tilde{\eta}\left(f, \tau^{\prime} f\right)\right\rangle \\
& -\left\langle\tilde{\eta}(f, \tau f)-\tilde{\eta}\left(\tau^{-1} f, f\right), \tilde{\eta}\left(f, \tau^{\prime} f\right)-\tilde{\eta}\left(\tau^{\prime-1} f, f\right)\right\rangle .
\end{aligned}
$$

Comme $\tau$ et $\tau^{\prime}$ commutent à $\Gamma$, ces trois fonctions sont $\Gamma$-invariantes. Aussi, comme l'action de $\Gamma$ est proprement discontinue, $\tau$ et $\tau^{\prime}$ définissent des bijections du quotient qui préservent la mesure $\mu$ induite sur $\Gamma \backslash F$. Comme les fonctions $u$ et $v$ sont dans $L^{1}(\Gamma \backslash F, \mu)$, il vient

$$
\begin{gathered}
\int_{\Gamma \backslash F}\left(u-u \circ \tau^{\prime}\right)(f) \mathrm{d} \mu(f)=0, \\
\int_{\Gamma \backslash F}(v \circ \tau-v)(f) \mathrm{d} \mu(f)=0,
\end{gathered}
$$

d'où

$$
\int_{\Gamma \backslash F} Q_{f}(\eta) \mathrm{d} \mu(f)=0 .
$$

\subsection{Réduction à une somme finie.}

Il reste à vérifier que, pour tout sommet $s$ de $X$, l'intégrale

$$
\int_{\mathcal{F}_{s}} Q_{f}(\eta) \mathrm{d} \mu(f)
$$

est proportionnelle à l'expression $Q_{s}(D \eta)$ qui figure dans la formule de Garland. On ramène d'abord cette intégrale à une somme finie. 
Pour chaque $f \in F$, l'expression $Q_{f}(\eta)$ ne fait intervenir que les six voisins du point base $s$ dans le plat $f\left(\pi_{0}\right)$, autrement dit, un 6-cycle marqué $\xi$ du link $\ell k(s)$. Il vient

$$
\int_{\mathcal{F}_{s}} Q_{f}(\eta) \mathrm{d} \mu(f)=\sum_{\text {6-cycles en } s} \mu\left(\mathcal{F}_{\xi}\right) Q_{\xi}(\eta),
$$

où

$$
\begin{aligned}
Q_{\xi}(\eta)=\langle & \left.\eta\left(y_{3}, y_{2}\right)-\eta\left(s, y_{1}\right), \eta\left(y_{1}, y_{2}\right)-\eta\left(s, y_{3}\right)\right\rangle \\
& -\left\langle\eta\left(s, y_{1}\right)+\eta\left(s, y_{4}\right), \eta\left(s, y_{3}\right)+\eta\left(s, y_{6}\right)\right\rangle .
\end{aligned}
$$

Comme la mesure $\mu\left(\mathcal{F}_{\xi}\right)$ ne dépend pas de $\xi$, et comme $\mu\left(\mathcal{F}_{s}\right)=1$, l'intégrale est simplement la moyenne arithmétique des $Q_{\xi}(\eta)$. On trouve

$$
\int_{\mathcal{F}_{s}} Q_{f}(\eta) \mathrm{d} \mu(f)=\frac{1}{N} \sum_{\xi} Q_{\xi}(\eta)
$$

où $N$, le nombre de 6 -cycles marqués, vaut $N=2 q^{3}(q+1)\left(q^{2}+q+1\right)$.

\subsection{Sommation sur les 6-cycles.}

On écrit maintenant la contribution d'un 6-cycle $\xi=\left\{y_{1}, \cdots, y_{6}\right)$ en fonction de $D \eta=(u, v)$. Par définition,

$$
\begin{aligned}
u(y) & =\eta(s, y), \\
v(y, z) & =\mathrm{d} \eta(s, y, z)=u(y)+\eta(y, z)-u(z) .
\end{aligned}
$$

Il vient

$$
\begin{aligned}
Q_{\xi}(\eta)=\left\langle v\left(y_{3}, y_{2}\right)+u\left(y_{2}\right)-u\left(y_{1}\right)-u\left(y_{3}\right)\right. & \\
v\left(y_{1}, y_{2}\right) & \left.+u\left(y_{2}\right)-u\left(y_{1}\right)-u\left(y_{3}\right)\right\rangle \\
& -\left\langle u\left(y_{1}\right)+u\left(y_{4}\right), u\left(y_{3}\right)+u\left(y_{6}\right)\right\rangle .
\end{aligned}
$$

Deux termes de cette expression qui diffèrent par une permutation circulaire de $\{1, \cdots, 6\}$ donnent la même valeur après sommation sur tous les cycles. Or chacun des 20 termes de l'expression ci-dessus coïncide après permutation circulaire avec l'un des 6 suivants

$$
\begin{array}{ll}
A=\left\langle v\left(y_{1}, y_{2}\right), v\left(y_{3}, y_{2}\right)\right\rangle, & B=\left\langle v\left(y_{1}, y_{2}\right), u\left(y_{1}\right)\right\rangle, \\
C=\left\langle u\left(y_{1}\right), v\left(y_{2}, y_{3}\right)\right\rangle, & D=\left\langle u\left(y_{1}\right), u\left(y_{1}\right)\right\rangle, \\
E=\left\langle u\left(y_{1}\right), u\left(y_{2}\right)\right\rangle, & F=\left\langle u\left(y_{1}\right), u\left(y_{3}\right)\right\rangle .
\end{array}
$$

TOME $126-1998-\mathrm{N}^{\circ} 1$ 
Par conséquent,

$$
\sum_{\xi} Q_{\xi}(\eta)=\sum_{\xi} A-4 \sum_{\xi} B+2 \sum_{\xi} C+3 \sum_{\xi} D-6 \sum_{\xi} E+0 \sum_{\xi} F
$$

\subsection{Calcul des sommes partielles.}

Soit $L$ le link en $s$. On note $\Sigma(0)$ (resp. $\Sigma(1)$ ) l'ensemble des sommets (resp. des couples de points voisins) dans $L$. Étant donné $y \in L$, on note $V(y)$ l'ensemble de ses voisins. On rappelle que, comme $L$ est un plan projectif, tout chemin de longueur 4 dans $L$ se prolonge uniquement en un 6-cycle de $L$.

Calcul de $\sum_{\xi} A$. - Étant donné un sommet $y$ de $L$ et deux sommets distincts $x$ et $z$ voisins de $y$, il existe autant de 6 -cycles $\xi$ tels que $x=y_{1}$, $y=y_{2}, z=y_{3}$ que de choix d'un quatrième point $y_{4}$ parmi les voisins de $z$ distincts de $y$. Il y a donc $q$ choix. Il vient :

$$
\begin{aligned}
\sum_{\xi} A= & q \sum_{(x, y) \in \Sigma(1)}\left\langle v(x, y), \sum_{\substack{z \in V(y) \\
z \neq x}}-v(y, z)\right\rangle \\
= & q \sum_{(x, y) \in \Sigma(1)}\left\langle v(x, y),-v(x, y)+\frac{1}{2} \delta v(y)\right\rangle \\
= & -q\langle v, v\rangle+q \sum_{y \in \Sigma(0)}\left\langle\frac{1}{2} \delta v(y), \sum_{x \in V(y)} v(x, y)\right\rangle \\
= & -q\langle v, v\rangle+\frac{1}{4} q\langle\delta v, \delta v\rangle .
\end{aligned}
$$

Calcul de $\sum_{\xi} B$. - Étant donné un couple de voisins $(x, y)$, il y a $q^{2}$ manières de construire un 6-cycle tel que $y_{1}=x$ et $y_{2}=y$. Par conséquent,

$$
\begin{aligned}
\sum_{\xi} B & =q^{2} \sum_{x \in \Sigma(0)}\left\langle u(x), \sum_{y \in V(x)} v(x, y)\right\rangle \\
& =q^{2} \sum_{x \in \Sigma(0)}\left\langle u(x),-\frac{1}{2} \delta v(x)\right\rangle \\
& =-\frac{1}{2} q^{2}\langle u, \delta v\rangle .
\end{aligned}
$$

Calcul de $\sum_{\xi} C$. - De nouveau, il y a $q$ manières de refermer un 3- 
chemin en un 6-cycle, donc :

$$
\begin{aligned}
\sum_{\xi} C & =q \sum_{(x, y) \in \Sigma(1)}\left\langle u(x), \sum_{\substack{z \in V(y) \\
z \neq x}} v(y, z)\right\rangle \\
& =q \sum_{(x, y) \in \Sigma(1)}\left\langle u(x),-\frac{1}{2} \delta v(y)-v(y, x)\right\rangle \\
& =q \sum_{(x, y) \in \Sigma(1)}\langle u(x), v(x, y)\rangle-q \sum_{y \in \Sigma(0)}\left\langle\frac{1}{2} \delta v(y), \sum_{x \in V(y)} u(x)\right\rangle \\
& =-\frac{1}{2} q\langle u+M u, \delta v\rangle,
\end{aligned}
$$
où on a noté $M u(y)=\sum_{x \in V(y)} u(x)$. D'autre part, pour tout sommet $x$
de $L$,

$$
\delta \mathrm{d} u(x)=-2 \sum_{y \in V(x)} u(y)-u(x)=-2 M u(x)+2(q+1) u(x) .
$$

Donc $M=-\frac{1}{2} \delta \mathrm{d}+q+1$ et

$$
\sum_{\xi} C=-\frac{1}{2} q(q+2)\langle u, \delta v\rangle+\frac{1}{4} q\langle\delta \mathrm{d} u, \delta v\rangle .
$$

Clairement,

$$
\sum_{\xi} D=q^{2}(q+1)\langle u, u\rangle
$$

et

$$
\begin{aligned}
\sum_{\xi} E & =q^{2}\langle u, M u\rangle \\
& =-\frac{1}{2} q^{2}\langle u, \delta \mathrm{d} u\rangle+q^{2}(q+1)\langle u, u\rangle \\
& =-\frac{1}{2} q^{2}\langle\mathrm{~d} u, \mathrm{~d} u\rangle+q^{2}(q+1)\langle u, u\rangle .
\end{aligned}
$$

Il vient

$$
\begin{aligned}
\sum_{\xi} Q_{\xi}(\eta) & =-q\langle v, v\rangle+\frac{1}{4} q\langle\delta v, \delta v\rangle+q(q-2)\langle u, \delta v\rangle \\
& +\frac{1}{2} q\langle\delta \mathrm{d} u, \delta v\rangle-3 q^{2}(q+1)\langle u, u\rangle+3 q^{2}\langle\mathrm{~d} u, \mathrm{~d} u\rangle .
\end{aligned}
$$

Ceci achève la preuve de la proposition 11 .

TOME $126-1998-\mathrm{N}^{\circ} 1$ 


\section{La mesure de Liouville sur l'espace des plats marqués}

\subsection{Préliminaires sur les immeubles triangulaires.}

Rappelons les propriétés fondamentales des immeubles triangulaires.

ThÉORÈme 1 (J. Tits, voir [T1]). - Soit $X$ un complexe simplicial de dimension 2, simplement connexe, dont les links sont des plans projectifs finis. Alors $X$ est à courbure négative ou nulle au sens d'Alexandrov. Décidons d'appeler appartements les sous-complexes isométriques au plan euclidien. Alors $X$ muni de cette famille d'appartements est un immeuble, en particulier, deux simplexes de $X$ sont contenus dans un même appartement.

Il résulte de la propriété de courbure négative ou nulle que $X$ est contractile, et que deux points de $X$ sont reliés par un unique segment géodésique.

Dans un polyèdre localement fini à courbure négative ou nulle, les géodésiques ont une caractérisation locale, i.e., qui se lit dans les links. On munit chaque link de sa métrique sphérique, de sorte que, si $y$ et $y^{\prime} \in \ell k(s)$ sont contenus dans un même simplexe, leur distance sph-dist $\left(y, y^{\prime}\right)$ est l'angle en $s$ entre les segments $s y$ et $s y^{\prime}$.

LEMME 15 (caractérisation des géodésiques, voir $[\mathrm{BH}]$ ). - Une courbe dans $X$ est une géodésique si et seulement si

(a) dans chaque simplexe, c'est un segment de droite;

(b) lorsqu'elle passe d'un simplexe à un autre en un point $x$ intérieur $\grave{a}$ une arête de $X$, l'angle d'incidence est égal à l'angle de sortie;

(c) lorsqu'elle passe d'un simplexe à un autre en un sommet s, ses directions d'incidence et de sortie sont des points du link $\ell k(s)$ situés à distance sphérique $\geq \pi$.

Enfin, nous aurons besoin d'un peu de combinatoire. On appelle $k$ chemin dans un graphe une suite $x_{1}, \ldots, x_{k}$ de sommets distincts tels que $\left(x_{i}, x_{i+1}\right)$ soit une arête. Si de plus $\left(x_{k}, x_{1}\right)$ est une arête, on parle de $k$-cycle marqué. Un $k$-cycle est le sous-complexe sous-jacent à un $k$-cycle marqué.

LEMME 16. - Soit $L$ le graphe d'incidence d'un plan projectif fini d'ordre $q$. Le nombre d'arêtes dans $L$ est $A=(q+1)\left(q^{2}+q+1\right)$, le nombre de 6-cycles contenant une arête donnée est $\alpha=q^{3}$, le nombre de 6-cycles contenant un 3-chemin donné est $\beta=q$.

On compte d'abord les points de $\mathcal{P}$. Ayant fixé un point $p_{0}$, les $q+1$ droites passant par $p_{0}$ forment une partition de $P \backslash\left\{p_{0}\right\}$ en ensembles de $q$ 
éléments, d'où $\# \mathcal{P}=q^{2}+q+1$. Ensuite, $A$ est le nombre de drapeaux $(p, \ell)$ où $p$ est un point de la droite $\ell$, donc $A=(q+1)\left(q^{2}+q+1\right)$.

Enfin, tout 4-chemin se prolonge en un unique 6-cycle marqué. Par conséquent, le nombre de 6-cycles contenant une arête est égal au nombre de 4-chemins possibles issus d'une arête orientée, d'où $\alpha=q^{3}$. On a de même $\beta=q$.

\subsection{Preuve de la proposition 14.}

La preuve de la proposition 14 occupe le reste de ce paragraphe.

\subsection{Bissecteurs et losanges.}

On appelle bissecteur de taille $n$ un segment géodésique reliant deux sommets de $X$ et qui partage en deux parties égales les simplexes qu'il traverse. La taille $n$ est la moitié du nombre de simplexes traversés, donc la longueur est $n \sqrt{3}$. L'enveloppe convexe d'un bissecteur est l'image d'un plongement isométrique d'un losange du plan euclidien, elle est partagée en deux par $b$. Une orientation de ce losange est le choix d'une des deux moitiés, qu'on appelera moitié droite de $b$.

Un bissecteur orienté $b$ a une origine $o(b)$, une extrémité $e(b)$. Si sa taille est paire, il a un milieu $m(b)$. Si sa taille $n=2 k-1$ est impaire, on appelle abusivement milieu de $b$ et on note $m(b)$ le point de $b$ situé à distance $k \sqrt{3}$ de $e(b)$.

Soit $b$ un bissecteur orienté de taille au moins 2. Le link en $m(b)$ de son enveloppe convexe est un 6-cycle, qu'on peut orienter de deux façons. Un bissecteur marqué est la donnée d'un bissecteur orienté $b$ et d'une orientation du link en $m(b)$ de son enveloppe convexe. Une fois cette orientation choisie, il y a une seule manière de numéroter $y_{1}, \ldots, y_{6}$ les points du link de sorte que la direction de sortie de $b$ en $m(b)$ soit le milieu de l'arête $\left(y_{6}, y_{1}\right)$. Par conséquent, se donner un bissecteur marqué, c'est se donner un bissecteur orienté et un 6-cycle marqué, le link de son enveloppe convexe. On doit penser à un bissecteur marqué comme à un germe de plat marqué.

Un bissecteur marqué peut-être raccourci de deux façons. On note $r(b)$ le bissecteur d'origine $o(b)$ et d'extrémité le point de $b$ situé à distance $\sqrt{3}$ de $e(b)$. De même, on note $r^{\prime}(b)$ le bissecteur d'origine le point de $b$ situé à distance $\sqrt{3}$ de $o(b)$ et d'extrémité $e(b)$. L'orientation des links de $b$ induit une orientation des links de $r(b)$ et de $r^{\prime}(b)$.

Dans le plan triangulé de référence $\pi_{0}$, on fixe pour tout $n$ un bissecteur marqué $b_{n}$. Sa taille est $n$, son milieu est $O$, sa direction est celle de la translation $\tau_{0}\left(\tau_{0}^{\prime}\right)^{-1}$, son link est orienté par le choix de l'ordre $y^{1}, \cdots, y^{6}$.

Soit $f: \pi_{0} \rightarrow X$ un plat marqué et $b$ un bissecteur marqué de taille $n$.

TOME $126-1998-\mathrm{N}^{\circ} 1$ 
On dit que $f$ contient $b$ (et on note $b \subset p$ ou bien $p \in \mathcal{F}_{b}$ ) si $b=f\left(b_{n}\right)$, et si $f$ préserve l'orientation des segments et des links. Chaque plat marqué $f$ contient donc un unique bissecteur marqué $b(f, n)$ de taille $n$ (la convention d'orientation est là pour cela). Lorsque $n$ tend vers l'infini, les ensembles $\mathcal{F}_{b(f, 2 n)}$ forment un système fondamental de voisinages de $f$ dans $F$.

\subsection{Construction de la mesure $\mu$.}

Soient $b$ et $b^{\prime}$ deux bissecteurs marqués. On rappelle que $\mathcal{F}_{b}$ est l'ensemble des plats marqués contenant $b$. Les ensembles $\mathcal{F}_{b}$ et $\mathcal{F}_{b^{\prime}}$ se rencontrent seulement si l'un est contenu dans l'autre, et c'est le cas seulement si $b \subset b^{\prime}$ ou $b^{\prime} \subset b$. Par conséquent, pour chaque point $s$ de $X$, et pour chaque $n$, lorsque $b$ décrit les bissecteurs marqués de longueur $2 n$ et de milieu $s$, les $\mathcal{F}_{b}$ forment une partition de l'ensemble $\mathcal{F}_{s}$ des plats marqués en $s$, partition de plus en plus fine. On note $B_{s, 2 n}$ le nombre de ces bissecteurs marqués. On définit une mesure de probabilité $\mu$ sur les boréliens de $\mathcal{F}_{s}$ en décidant que $\mu\left(\mathcal{F}_{b}\right)=1 / B_{s, 2 n}$.

\subsection{Calcul de $\boldsymbol{B}_{\boldsymbol{s}, \boldsymbol{n}}$.}

On compte d'abord les bissecteurs marqués de longueur 2 et de milieu $s$. Comptons d'abord les candidats possibles pour les directions d'entrée et de sortie en $s$. Il y a $A=(q+1)\left(q^{2}+q+1\right)$ choix pour la direction d'entrée (milieu d'une arête $a$ ), puis $\alpha=q^{3}$ choix pour la direction de sortie (exactement un point à distance sphérique $\pi$ dans chaque 6 -cycle contenant $a$ ), soit $A \alpha$ possibilités pour un segment symétrique $\left[w, w^{\prime}\right]$ de longueur $\sqrt{3}$ centré en $s$.

Ensuite, on prolonge le segment $\left[w, w^{\prime}\right]$ au-delà de $w$ et de $w^{\prime}$. Il y a, à chaque extrémité, autant de choix que de 2-simplexes partageant une face avec un 2-simplexe donné, soit $q$. On trouve $A \alpha q^{2}$ bissecteurs orientés.

Enfin, le link en $s$ peut être orienté de deux façons. On trouve $2 A \alpha q^{2}=$ $2(q+1)\left(q^{2}+q+1\right) q^{5}$ bissecteurs marqués de taille 2 de milieu $s$.

Ensuite, étant donné un bissecteur marqué $b$ de milieu $s$, de longueur $n$, on compte le nombre de bissecteurs marqués $b^{\prime}$ de milieu $s$, de longueur $n+1$, qui le contiennent, i.e., tels que $b=r\left(b^{\prime}\right)$ (si $n$ est impair) et $b=r^{\prime}\left(b^{\prime}\right)$ (si $n$ est pair).

L'orientation de $b$ et celle du link de $b$ déterminent celles de $b^{\prime}$ et du link de $b^{\prime}$, donc on peut oublier les orientations. Si $n$ est pair, il faut compter les prolongements de $b$ au-delà de $o(b)$. Or $b$ détermine un point $y$ du link en $o(b)$ et il faut d'abord compter les points à distance sphérique $\pi$ de $y$. Il y en a exactement un dans chaque 6-cycle contenant $y$, soit $\alpha$ au total. Ceci compte les prolongements géodésiques sur une longueur $\frac{1}{2} \sqrt{3}$. Il reste 
à traverser un second simplexe, il y a $q$ choix. On trouve le nombre $\alpha q$.

Dans le cas où $n$ est impair, il suffit de remplacer l'origine $o(b)$ par l'extrémité $e(b)$.

On trouve

$$
B_{s, n}=2 A q(\alpha q)^{n-1}=2(q+1)\left(q^{2}+q+1\right) q^{4 n-3} .
$$

La mesure $\mu_{n}$ de l'ensemble $\mathcal{F}_{b}$ ( $b$ de taille $n$ ), est l'inverse de ce nombre, qui ne dépend que de la taille. Par conséquent, la mesure $\mu$ est invariante par tout automorphisme de $X$.

\subsection{Calcul de la mesure de $\mathcal{F}_{\boldsymbol{\xi}}$.}

Soit $\xi=\left(y_{1}, \cdots, y_{6}\right)$ un 6-cycle marqué du link $\ell k(s)$. On rappelle que $\mathcal{F}_{\xi}$ est l'ensemble des plats marqués en $s$ qui contiennent $\xi$. On décompose $\mathcal{F}_{\xi}$ en une réunion disjointe d'ensembles $\mathcal{F}_{b}$ où $b$ décrit certains bissecteurs marqués de taille 2 et de milieu $s$.

Notons $w\left(\right.$ resp. $\left.w^{\prime}\right)$ le milieu de l'arête $\left(y_{3}, y_{4}\right)$ (resp. $\left.\left(y_{1}, y_{6}\right)\right)$. Notons $E$ l'ensemble des bissecteurs marqués $b$ de taille 2 tels que

- $b$ contient le segment géodésique $\left[w, w^{\prime}\right]$ (orientation comprise);

- l'orientation choisie du link de l'enveloppe convexe de $b$ est celle de $\xi$.

Soit $f: \pi_{0} \rightarrow X$ un plat marqué qui contient $\xi$. Le plat $f$ contient un unique bissecteur marqué $b(f, 2)$ de taille 2 et de milieu $s$, et celui-ci contient le segment géodésique $\left[w, w^{\prime}\right]$ (orientation comprise). L'orientation induite par $b(f, 2)$ sur $\xi$ est la bonne, donc $b(f, 2) \in E$. Inversement, si $b \in E$, tout plat marqué contenant $b$ contient $\xi$ en déterminant la bonne orientation. Par conséquent

$$
\mathcal{F}_{\xi}=\coprod_{b \in E} \mathcal{F}_{b}
$$

Il reste à compter les bissecteurs autorisés. Ils s'obtiennent en prolongeant $\left[w, w^{\prime}\right]$ au-delà de chaque extrémité par la hauteur d'un simplexe. Il y a $q$ choix à chaque extrémité, donc $q^{2}$ choix pour $b$. On conclut que $\mu\left(\mathcal{F}_{\xi}\right)=q^{2} \mu_{2}$ est indépendant du choix de $\xi$.

\subsection{Calcul de la mesure de $\tau^{-1}\left(\mathcal{F}_{b}\right)$.}

On vérifie l'invariance de $\mu$ par l'action de $\mathbb{Z} \oplus \mathbb{Z}$. Il suffit de vérifier que, pour tout bissecteur marqué $b$ de taille impaire assez grande, $\mu\left(\tau^{-1}\left(\mathcal{F}_{b}\right)\right)=\mu\left(\mathcal{F}_{b}\right)$. La vérification est identique pour $\tau^{\prime}$.

Soit $b$ un bissecteur marqué de taille $2 n-1$, où $n \geq 2$. On va décomposer $\tau^{-1}\left(\mathcal{F}_{b}\right)$ en une réunion disjointe d'ensembles de la forme $\mathcal{F}_{b^{\prime}}$, où les $b^{\prime}$ sont de taille $2 n$.

TOME $126-1998-\mathrm{N}^{\circ} 1$ 
Soit $b_{2 n}$ le bissecteur modèle du plan $\pi_{0}$. Son enveloppe convexe est un losange. L'intersection de ce losange et de $\tau_{0}\left(b_{2 n}\right)$ est un bissecteur $\tau_{r}\left(b_{2 n}\right)$ de taille $2 n-1$, parallèle et de même orientation que $b_{2 n}$. Cette construction s'étend à tout bissecteur marqué $b^{\prime}$ de taille $2 n$. Elle a la propriété suivante : pour tout plat marqué $f$ contenant $b^{\prime}, \tau(f)\left(r\left(b_{2 n}\right)\right)=\tau_{r}\left(b^{\prime}\right)$, milieu et orientation compris. En particulier, si $f \in \mathcal{F}_{b^{\prime}}$, alors $\tau(f) \in \mathcal{F}_{\tau_{r}\left(b^{\prime}\right)}$. Inversement, si un plat marqué $f$ satisfait $\tau(f) \in \mathcal{F}_{b}$, alors $f\left(\pi_{0}\right)$ contient un unique bissecteur marqué $b^{\prime}$ de taille $2 n$ tel que $\tau_{r}\left(b^{\prime}\right)=b$. Par conséquent, $\tau^{-1}\left(\mathcal{F}_{b}\right)$ est la réunion disjointe des $\mathcal{F}_{b^{\prime}}$ où $b^{\prime}$ décrit les bissecteurs marqués de taille $2 n$ tels que $\tau_{r}\left(b^{\prime}\right)=b$. Il ne reste qu'à compter ces bissecteurs.

On remarque que, si $\tau_{r}\left(b^{\prime}\right)=b$, alors le bissecteur marqué $b^{\prime \prime}=r^{\prime} r\left(b^{\prime}\right)$ de taille $2 n-2$ est contenu dans l'enveloppe convexe de $b$, et ne dépend que de $b$. En revanche, $r\left(b^{\prime}\right)$ dépend de $b^{\prime}$. On compte d'abord les possibilités pour $r\left(b^{\prime}\right)$, i.e., les bissecteurs $b^{\prime \prime \prime}$ de taille $2 n-1$ tels que $r^{\prime}\left(b^{\prime \prime \prime}\right)=b^{\prime \prime}$ et dont l'enveloppe convexe contient $r(b)$. Il s'agit de prolonger $b^{\prime \prime}$ audelà de $o\left(b^{\prime \prime}\right)$, i.e., de choisir d'abord dans le link de $o\left(b^{\prime \prime}\right)$ un 6-cycle qui prolonge la trace du losange $\operatorname{Conv}(b)$ (c'est un 3-chemin $c$ ). D'après 16, il y a $\beta=q$ choix. Chaque choix détermine un simplexe $\sigma$ dont une hauteur prolonge $b^{\prime \prime}$ en une géodésique. Ensuite, il faut choisir un simplexe partageant un côté avec $\sigma$, soit $q$ choix. On trouve donc $q^{2}$ possibilités pour $b^{\prime \prime \prime}$.

Le bissecteur $b^{\prime \prime \prime}$ étant fixé, on compte les bissecteurs marqués $b^{\prime}$ tels que $r\left(b^{\prime}\right)=b^{\prime \prime \prime}$ et dont l'enveloppe convexe contient $b$. Il s'agit de prolonger $b^{\prime \prime \prime}$ au-delà de $e\left(b^{\prime \prime \prime}\right)$, i.e., de choisir d'abord dans le link de $e\left(b^{\prime \prime \prime}\right)$ un 6-cycle qui prolonge la trace du losange $\operatorname{Conv}(b)$ (c'est un 3 -chemin $c^{\prime}$ ). Il y a $\beta=q$ choix. Chaque choix détermine un simplexe $\sigma^{\prime}$ dont une hauteur prolonge $b^{\prime \prime \prime}$ en une géodésique. Enfin, il reste à choisir un simplexe partageant un côté avec $\sigma^{\prime}$, soit $q$ choix. Le bissecteur $b^{\prime \prime \prime}$ étant fixé, on trouve $q^{2}$ possibilités pour $b^{\prime}$.

Il y a donc $q^{4}$ bissecteurs marqués $b^{\prime}$ tels que $\tau_{r}\left(b^{\prime}\right)=b$. On conclut que

$$
\mu\left(\tau^{-1}\left(\mathcal{F}_{b}\right)\right)=q^{4} \mu_{2 n}=\mu\left(\mathcal{F}_{b}\right),
$$

donc la mesure $\mu$ est invariante par la transformation $\tau$. La preuve est la même pour $\tau^{\prime}$. Ceci achève la preuve de la proposition 14 .

\section{Applications de la formule de Garland}

Elles découlent de la propriété de positivité suivante, dont la preuve est simple.

Proposition 17 (W. Feit, G. Higman, voir [FH]). - Lorsque L est 
le graphe d'incidence d'un plan projectif d'ordre $q$, la forme quadratique $Q_{L}(u)=\langle\mathrm{d} u, \mathrm{~d} u\rangle-(q+1)\langle u, u\rangle$ sur $C^{0}(L)$ a exactement une direction négative, celle des fonctions constantes.

Lemme 18. - Soit $X$ un immeuble triangulaire, $\Gamma$ un groupe discret d'automorphismes de $X, \rho$ une représentation unitaire de $\Gamma$. Il existe une constante $C$ indépendante de la représentation $\rho$ telle que pour toute 1 cochaîne $L^{2}$ sur $\Gamma \backslash X$ tordue par $\rho$,

$$
\|\eta\|^{2} \leq C\left(\|\delta \alpha\|^{2}+\|\mathrm{d} \alpha\|^{2}\right)
$$

Preuve. - Soit $\eta$ une 1-cochaîne scalaire sur $X$. Soit $s$ un sommet de $X$. Posons $D \eta=(u, v)$ où $u$ est une fonction sur les sommets du link $L=\ell k(s)$ et $v$ un 1-cocycle sur $L$. D'après la proposition 17 , l'expression $Q_{s}(D \eta)$ est une forme quadratique sur $C^{0}(L) \oplus C^{1}(L)$ qui est définie positive sur le sous-espace des couples $(u, v)$ tels que $v=0$ et $u$ soit orthogonal aux fonctions constantes. Autrement dit, si on note $\tau u$ la somme des valeurs de $u$ sur les sommets de $L$, il existe des constantes $C^{\prime}$ et $C^{\prime \prime}$ ne dépendant que de l'ordre de $X$ telles que

$$
\langle u, u\rangle \leq C^{\prime}\left(|\tau u|^{2}+\langle v, v\rangle\right)+C^{\prime \prime} Q_{s}(D \eta) .
$$

La même inégalité reste vraie, avec les mêmes constantes, pour une cochaîne à valeurs dans un espace de Hilbert $\mathcal{H}_{\rho}$. Supposons $\eta$ équivariante sous un groupe discret $\Gamma$ et de carré intégrable modulo $\Gamma$. En sommant sur les sommets de $\Gamma \backslash X$, il vient, avec la formule de Garland,

$$
2\|\eta\|^{2} \leq C^{\prime}\left(\|\delta \alpha\|^{2}+3\|\mathrm{~d} \alpha\|^{2}\right)
$$

COROllaire 19. - Sous les mêmes hypothèses, on obtient des conséquences sur la cohomologie $L^{2}$ de $\Gamma \backslash X$ tordue par $\rho$. L'espace $L^{2} H^{1}(\Gamma \backslash X, \rho)$ est nul et l'espace $L^{2} H^{2}(\Gamma \backslash X, \rho)$ est séparé.

Corollaire 20. - Soit $X$ un immeuble triangulaire, $\Gamma$ un groupe discret cocompact d'isométries de $X, \rho$ une représentation unitaire de $\Gamma$. Alors $H^{1}(\Gamma, \rho)=0$ et $H^{2}(\Gamma, \rho)$ est séparé. En particulier, $\Gamma$ possède la propriété $(\mathrm{T})$ de Kazhdan et est «big» au sens de J. Lott.

Remarque 21. - On trouvera dans [CMS] et [CMSZ] une approche directe de la propriété ( $\mathrm{T}$ ) pour certains groupes agissant sur des immeubles triangulaires. Cette approche fournit plus d'informations sur les représentations unitaires.

TOME $126-1998-\mathrm{N}^{\circ} 1$ 


\section{Cas des immeubles carrés}

Un immeuble carré est un immeuble euclidien modelé sur $\widetilde{A}_{1} \times \widetilde{A}_{1}$. Autrement dit, les appartements sont des plans euclidiens pavés par des carrés. La méthode du paragraphe 7 va permettre d'obtenir une nouvelle formule, dont on donnera une application (propriété $(\mathrm{T})$ pour les quotients de certains groupes discrets). La construction de la mesure invariante sur l'espace des plats marqués est facile, car un immeuble carré est le produit de deux arbres.

\subsection{Formule a la Garland pour un produit d'arbres.}

Soit $T$ un arbre régulier de valence $v$. Une géodésique marquée dans $T$ est une application simpliciale injective $g: \mathbb{R} \rightarrow T$ où la droite réelle est pavée par des segments de longueur 1 aux points entiers. L'espace $\mathcal{G}(T)$ des géodésiques marquées porte une action de $\mathbb{Z}$ (précomposition par les translations entières) et une mesure $\mathbb{Z}$-invariante $\mu$ définie comme suit. Pour chaque géodésique marquée $g$, l'ensemble des géodésiques marquées qui coincident avec $g$ sur l'intervalle $[0, n]$ a pour mesure $v^{-1}(v-1)^{1-n}$. Pour chaque sommet $s$ de $T$, la mesure du sous-ensemble des géodesiques marquées d'origine $s$ vaut 1 et la mesure du sous-ensemble des géodesiques marquées telles que $g([0,1])$ est une arête donnée vaut $1 / v$.

Soient $T_{1}$ et $T_{2}$ des arbres simpliciaux. Leur produit $X=T_{1} \times T_{2}$ est un complexe carré, et il est classique qu'on peut calculer la cohomologie sur un quotient de $X$ au moyen de cochaînes carrées, i.e., de fonctions sur l'ensemble des cellules orientées. Une 1-cochaîne carrée sur $X$ est une fonction $\eta$ sur l'ensemble des arêtes de $X$. Sa différentielle $\mathrm{d} \eta$ est une fonction sur l'ensemble des carrés. Le bord d'un carré orientée $\sigma$ est la somme de 4 arêtes orientées $a_{1}, a_{2}, a_{1}^{\prime}, a_{2}^{\prime}$ et

$$
\mathrm{d} \eta(\sigma)=\eta\left(a_{1}\right)+\eta\left(a_{2}\right)+\eta\left(a_{1}^{\prime}\right)+\eta\left(a_{2}^{\prime}\right) .
$$

DÉfinition 22. - Soit $\eta$ une 1-cochaîne sur $X=T_{1} \times T_{2}$ à valeurs dans un espace de Hilbert $\mathcal{H}$. On note $D \eta$ l'application qui à chaque carré orienté $\sigma$ de $X$ d'arêtes orientées $a_{1}, a_{2}, a_{1}^{\prime}, a_{2}^{\prime}$ (où $a_{1}$ et $a_{1}^{\prime}$ sont parallèles au premier facteur) associe le vecteur

$$
D \eta(\sigma)=\eta\left(a_{1}\right)+\eta\left(a_{1}^{\prime}\right)-\eta\left(a_{2}\right)-\eta\left(a_{2}^{\prime}\right) .
$$

On note

$$
\delta_{1} \eta(s)=\sum \eta\left(a_{1}\right)
$$

où la somme est étendue aux arêtes parallèles au premier facteur, d'origine $s$. 
Un plat marqué dans l'immeuble $X=T_{1} \times T_{2}$ est simplement un couple $f=\left(g_{1}, g_{2}\right)$ de géodésiques marquées $g_{1}$ de $T_{1}$ et $g_{2}$ de $T_{2}$.

Écrivons la formule de Bochner simpliciale dans un tel plat, pour un 1-cocycle carré $\eta$ à valeurs dans un espace de Hilbert $\mathcal{H}$. Le terme quadratique au sommet $s=\left(g_{1}(0), g_{2}(0)\right)$ fait intervenir les 6 arêtes orientées. Quatre d'entre elles bordent un carré $\sigma$. On les note

$$
\begin{array}{ll}
a_{1}=\left(g_{1}([0,1]), g_{2}(0)\right), & a_{1}^{\prime}=\left(g_{1}([1,0]), g_{2}(1)\right), \\
a_{2}=\left(g_{1}(0), g_{2}([1,0])\right), & a_{2}^{\prime}=\left(g_{1}(1), g_{2}([0,1])\right) .
\end{array}
$$

Les deux autres sont notées

$$
b_{1}=\left(g_{1}([0,-1]), g_{2}(0)\right), \quad b_{2}=\left(g_{1}(0), g_{2}([0,-1])\right)
$$

(attention, on a renversé leur orientation par rapport à la notation de 7.1). Alors

$$
\begin{aligned}
Q_{f}(\eta) & =\left\langle\eta\left(a_{1}^{\prime}\right)+\eta\left(a_{1}\right), \eta\left(a_{2}\right)+\eta\left(a_{2}^{\prime}\right)\right\rangle+\left\langle\eta\left(a_{1}\right)+\eta\left(b_{1}\right), \eta\left(a_{2}\right)+\eta\left(b_{2}\right)\right\rangle \\
& =-|D \eta(\sigma)|^{2}+\left\langle\eta\left(a_{1}\right)+\eta\left(b_{1}\right), \eta\left(a_{2}\right)+\eta\left(b_{2}\right)\right\rangle
\end{aligned}
$$

ne dépend que de $\sigma, b_{1}$ et $b_{2}$. On note $Q_{s}(\eta)$ la moyenne de $Q_{f}(\eta)$ sur le sous-ensemble des plats marqués d'origine $s$ (c'est-à-dire tels que $\left.s=\left(g_{1}(0), g_{2}(0)\right)\right)$. Alors

$$
\begin{aligned}
Q_{s}(\eta) & =\int_{s=\left(g_{1}(0), g_{2}(0)\right)} Q_{f}(\eta) \mathrm{d} \mu_{1}\left(g_{1}\right) \mathrm{d} \mu_{2}\left(g_{2}\right) \\
& =v_{1}^{-1} v_{2}^{-1}\left(\sum v_{1} v_{2}|D \eta(\sigma)|^{2}+\sum\left\langle\eta\left(a_{1}\right)+\eta\left(b_{1}\right), \eta\left(a_{2}\right)+\eta\left(b_{2}\right)\right\rangle\right)
\end{aligned}
$$

où la première somme est étendue à tous les carrés orientés ayant $s$ pour l'un de leur sommets et la seconde sur les quadruplets $\left(a_{1}, a_{2}, b_{1}, b_{2}\right)$ tels que $a_{1} \neq a_{2}$ et $b_{1} \neq b_{2}$. La seconde somme vaut

$$
\frac{1}{4} v_{1}\left(v_{1}-1\right) v_{2}\left(v_{2}-1\right)\left\langle\delta_{1} \eta(s), \delta_{2} \eta(s)\right\rangle .
$$

Supposons maintenant que $\eta$ est équivariant pour un groupe cocompact $\Gamma$ d'automorphismes de $X$. Lorsqu'on intègre sur l'espace $F$ des plats marqués (modulo $\Gamma$ ), il vient

$$
\begin{aligned}
\int_{\Gamma \backslash F} Q_{f}(\eta) \mathrm{d} \mu_{1} \mathrm{~d} \mu_{2} & =\sum_{s \in \Gamma \backslash X} Q_{s}(\eta) \\
& =4\|D \eta\|^{2}+\frac{1}{4}\left(v_{1}-1\right)\left(v_{2}-1\right)\left\langle\delta_{1} \eta, \delta_{2} \eta\right\rangle,
\end{aligned}
$$

et cette intégrale est nulle, pour la même raison qu'en 7.4. Comme on a $\delta=\delta_{1}+\delta_{2}$, il vient

$$
\text { TOME } 126-1998-\mathrm{N}^{\circ} 1
$$


Proposition 23 (formule «à la Garland》pour un immeuble carré). - Soient $T_{1}, T_{2}$ des arbres réguliers de valences $v_{1}$ et $v_{2}$. Soit $\Gamma$ un groupe discret cocompact d'automorphismes de $X=T_{1} \times T_{2}$. Soit $\rho$ une représentation unitaire de $\Gamma$. Soit $\eta$ un 1-cocycle sur $\Gamma \backslash X$ tordu par $\rho$. Alors

$$
\|\delta \eta\|^{2}=\left\|\delta_{1} \eta\right\|^{2}+\left\|\delta_{2} \eta\right\|^{2}+C\|D \eta\|^{2}
$$

où $C=32\left(v_{1}-1\right)^{-1}\left(v_{2}-1\right)^{-1}$.

\subsection{Groupes transitifs sur les sphères.}

Définition 24. - Un groupe $G$ agissant sur un espace métrique $(X, d)$ est dit transitif sur les sphères si pour tout point $x$ de $X$ et tout réel $r$, le stabilisateur $G_{x}$ agit transitivement sur la sphère $\partial B(x, r)$.

Le lemme suivant est dû à M. Burger et $\mathrm{S}$. Mozes [BM].

Lemme 25. - Soit $G$ un groupe qui agit simplicialement sur un arbre simplicial $T$ dont tout sommet a une valence au moins égale à 3 . On suppose que $G$ agit transitivement sur les sphères. Soit $N$ un sous-groupe distingué de $G$ qui n'a pas de point fixe global dans $T$. Alors pour tout sommet $x$ de $T$, le stabilisateur $N_{x}$ agit transitivement sur la sphère unité $\partial B(x, 1)$.

Preuve. - L'orbite $N x$ est une réunion de sphères centrées en $x$. En effet, soit $y \in N x$ situé à distance $r$ de $x$ et soit $y^{\prime}$ un autre sommet de $T$ situé à distance $r$ de $x$. Il existe $g \in G$ tel que $g x=x$ et $g y=y^{\prime}$. Alors $g N x=N g x=N x$ donc $y^{\prime} \in N x$.

Soient $(x, y)$ et $\left(x, y^{\prime}\right)$ deux arêtes orientées distinctes d'origine $x$. Comme tout sommet de $T$ a une valence $>2$, il existe un rayon géodésique infini $\rho$ d'origine $x$ qui ne contient ni $y$ ni $y^{\prime}$. Comme $N x \neq\{x\}, N x$ contient au moins une sphère de rayon non nul. Celle-ci rencontre $\rho$. On peut donc choisir $n \in N$ tel que $n x \neq x$ et $n x \in \rho$. Notons $z$ celui des deux points $n x$ et $n y$ qui est le plus éloigné de $x$. Alors la distance de $z$ au segment géodésique $\left[y, y^{\prime}\right]$ est atteinte en $x$, donc $d(z, y)=d\left(z, y^{\prime}\right)$. Soit $g \in G$ tel que $g z=z$ et $g y=y^{\prime}$. Alors $g x=x, g n x=n x$ et $g n y=y$. En effet, les segments géodésiques minimisants $[z, y]$ et $\left[z, y^{\prime}\right]$ passent tous deux par $x, n x$ et $n y$. Par conséquent, $g$ fixe l'arête orientée $(n x, n y)$ et envoie $(x, y)$ sur $\left(x, y^{\prime}\right)$. Comme $G$ permute les orbites de $N$ dans l'ensemble $T^{(1)}$ des arêtes orientées de $T,\left(x, y^{\prime}\right)$ est dans l'orbite de $(x, y)$, i.e., il existe $n^{\prime} \in N$ tel que $n^{\prime} x=x$ et $n^{\prime} y=y^{\prime}$.

Lemme 26. - Soit $\Gamma$ un groupe discret cocompact d'automorphismes de $X=T_{1} \times T_{2}$ dont les projections dans $\operatorname{Aut}\left(T_{1}\right)$ et $\operatorname{Aut}\left(T_{2}\right)$ agissent transitivement sur les sphères. Soit $N$ un sous-groupe distingué non trivial 
de $\Gamma$. Il existe une constante $C=C(N)$ ayant la propriété suivante. Soit $\rho$ une représentation unitaire de $\Gamma / N$. Pour tout 1 -cocyle $\Gamma$-équivariant $\eta$ sur $X$ à valeurs dans $\mathcal{H}_{\rho}$ on a l'inégalité

$$
\|\eta\|^{2} \leq C\left(\left\|\delta_{1} \eta\right\|+\left\|\delta_{2} \eta\right\|+\|D \eta\|^{2}\right) .
$$

Preuve. - D'après $[\mathrm{BM}]$, tout élément de $\Gamma$, et donc de $N$, agit non trivialement sur chacun des facteurs. Rappelons brièvement l'argument. Soit $H$ l'adhérence de l'image de $\Gamma$ dans $\operatorname{Aut}\left(T_{1}\right)$. Soit $\Lambda \subset \Gamma$ le sousgroupe des éléments qui agissent trivialement sur le second facteur. C'est un sous-groupe distingué dont l'image dans Aut $\left(T_{1}\right)$ est discrète, donc $\Lambda$ est discret et distingué dans $H$. $H$ permute les orbites de $\Lambda$ dans l'ensemble des bouts $\partial T_{1}$. Or $H$ est 2-transitif sur $\partial T_{1}$. Les orbites étant dénombrables, elles sont distinctes de $\partial T_{1}$, donc elles ont toutes un seul élément. Comme $\Lambda$ agit trivialement sur $\partial T_{1}$ donc sur $T_{1}, \Lambda=\{1\}$.

Montrons que $N$ n'a pas de point fixe global dans chaque facteur. S'il est non vide, l'ensemble des points fixes globaux de $N$ sur l'arbre $T_{i}$ contient une orbite de $\Gamma$, donc son enveloppe convexe est $T_{i}$ tout entier, et $N$ agit trivialement sur $T_{i}$, contradiction. Par conséquent, le couple $(\Gamma, N)$ satisfait aux hypothèses du lemme 25 .

Fixons un domaine fondamental fini $U$ pour $\Gamma$ dans $X^{(0)}$. Pour chaque sommet $s=\left(s_{1}, s_{2}\right) \in U$, choisissons un sous-ensemble fini $F_{s} \subset N$ tel que si $y, y^{\prime}$ sont des sommets de l'arbre $T_{1}$ situés à distance 1 de $s_{1}$, il existe $n \in F_{s}$ tel que $n s_{1}=s_{1}$ et $n y=y^{\prime}$ (et de même dans l'arbre $T_{2}$ ). Soit $M$ le maximum de la distance de $s$ à $n s$ pour $s \in U$ et $n \in F_{s}$.

Étant données deux arêtes $a$ et $a^{\prime}$ issues de $s$ et parallèles au premier facteur, on compare $\eta(a)$ et $\eta\left(a^{\prime}\right)$. Choisissons $n \in F_{s}$ tel que $n \operatorname{pr}_{1}\left(a^{\prime}\right)=$ $\operatorname{pr}_{1}(a)$. Comme $n$ agit trivialement sur $\mathcal{H}_{\rho}, \eta\left(n a^{\prime}\right)=\eta\left(a^{\prime}\right)$. Comme les arêtes $n a^{\prime}$ et $a$ ont la même projection, elles bordent une rangée de carrés en nombre au plus $M$. On a donc

$$
\left|\eta\left(n a^{\prime}\right)-\eta(a)\right| \leq M \sup \left\{\left|D \eta\left(s^{\prime}\right)\right| ; s^{\prime} \in U\right\} .
$$

Notons $v_{1}$ la valence du sommet $s_{1}$ dans $T_{1}$. De l'identité

$$
v_{1} \eta(a)=\delta_{1} \eta(s)+\sum \eta(a)-\eta\left(a^{\prime}\right)
$$

on tire

$$
\sum|\eta(a)|^{2} \leq \mathrm{C}^{\mathrm{te}}\left(\left|\delta_{1} \eta(s)\right|^{2}+\sum_{s^{\prime} \in U}\left|D \eta\left(s^{\prime}\right)\right|^{2}\right) .
$$

On a une estimation analogue pour les arêtes parallèles au second facteur. Il vient

$$
|\eta|(s)^{2} \leq \mathrm{C}^{\mathrm{te}}\left(\left|\delta_{1} \eta(s)\right|^{2}+\left|\delta_{2} \eta(s)\right|^{2}+\sum_{s^{\prime} \in U}\left|D \eta\left(s^{\prime}\right)\right|^{2}\right),
$$

TOME $126-1998-\mathrm{N}^{\circ} 1$ 
où la constante ne dépend du sous-groupe distingué $N$ qu'à travers la constante $M$. En sommant sur les sommets du domaine fondamental $U$, il vient

$$
\|\eta\|^{2} \leq \mathrm{C}^{\mathrm{te}}\left(\left\|\delta_{1} \eta\right\|^{2}+\left\|\delta_{2} \eta\right\|^{2}+\|D \eta\|^{2}\right)
$$

où il s'agit de normes $L^{2}$ sur $\Gamma \backslash X$.

Corollaire 27. - Soient $T_{1}, T_{2}$ des arbres réguliers de valences $>2$. Soit $\Gamma$ un groupe discret cocompact d'automorphismes de $X=T_{1} \times T_{2}$ dont les projections dans $\operatorname{Aut}\left(T_{1}\right)$ et $\operatorname{Aut}\left(T_{2}\right)$ agissent transitivement sur les sphères. Soit $N$ un sous-groupe distingué non trivial de $\Gamma$. Alors $\Gamma / N$ a la propriété $(T)$ de Kazhdan.

En effet, soit $\rho$ une représentation unitaire de $\Gamma$. Soit $\eta$ un 1-cocycle sur $\Gamma \backslash X$ tordu par $\rho$. Combinant la formule 23 et l'inégalité 26 , on trouve une inégalité de la forme $\|\eta\| \leq \mathrm{C}^{\text {te }}\|\delta \eta\|$ qui entraîne que $H^{1}(\Gamma, \rho)=0$.

REMARQUE 28. - Les groupes d'automorphismes d'un produit d'arbres qui satisfont l'hypothèse du corollaire 27 ont ils la propriété $\tau$ de [LZ] ? Cela revient à montrer que la constante $C(N)$ du lemme 26 est en fait indépendante du choix du sous-groupe distingué $N$, voir [L].

\section{BIBLIOGRAPHIE}

[B] Borel (A.). - Cohomologie de certains groupes discrets et laplaciens $p$-adiques [d'après H. Garland], Séminaire Bourbaki, exposé nº 437, 1973 .

[Ba] BARRe (S.). - Polyèdres finis de dimension 2 à courbure $\leq 0$ et de rang 2, Ann. Inst. Fourier, t. 45, 1995, p. 1037-1059.

[BB] Ballmann (W.), BRIN (M.). - Orbihedra of nonpositive curvature, Publ. Math., Institut Hautes Études Scientifiques, t. 82, 1995, p. 169-209.

[BH] Bridson (M.), Haefliger (A.). - CAT(0) spaces, livre à paraître.

[BM] Burger (M.), Mozes (S.). - Finitely presented groups and products of trees, C. R. Acad. Sci. Paris, t. 324, 1997, p. 747-752.

[BoS] Borel (A.), Serre (J.-P.). - Corners and arithmetic groups, Comment. Math. Helvet., t. 48, 1973, p. 436-491. 
[BS] Ballmann (W.), Swiatkowski (J.). - On $L^{2}$-cohomology and property $(T)$ for automorphism groups of cell complexes. - Preprint, Bonn, 1996.

[Bu] Burger (M.). - Kazhdan constants for $\mathrm{SL}(3, \mathbb{Z})$, J. Reine angew. Math., t. 385, 1991, p. 203-220.

[Br] Brown (K.). - Cohomology of groups, Graduate Texts in Mathematics, t. 87, Springer, Berlin, 1982.

[BW] Borel (A.), WAllach (N.). - Continuous cohomology, discrete subgroups and representations of reductive groups, Princeton University Press, Princeton, 1980.

[C] Corlette (K.). - Archimedean superrigidity and hyperbolic geometry, Ann. of Math., t. 135, 1992, p. 165-182.

[CMS] Cartwright (D.I.), Mlotkowski (W.), Steger (T.). - Property $(T)$ and $\widetilde{A}_{2}$ groups, Ann. Inst. Fourier, t. 44, 1993, p. 213-248.

[CMSZ] Cartwright (D.I.), Mantero (A.-M.), Steger (T.), Zappa (A.). - Groups acting simply transitively on the vertices of a building of type $\widetilde{A}_{2}$, Geom. Dedic., t. 47, I, p. 143-166, 1993; II, p. 167-223, 1993.

[DV] DE la Harpe (P.), Valette (A.). — La propriété $(T)$ de Kazhdan pour les groupes localement compacts, Astérisque, Soc. Math. de France, t. 175, 1989 .

[FH] Feit (W.), Higman (G.). - The nonexistence of certain generalized polygons, J. of Algebra, t. 1, 1964, p. 114-131.

[G] Garland (H.). - p-adic curvature and the cohomology of discrete subgroups of p-adic groups, Ann. of Math., t. 97, 1973, p. 375-423.

[GR] Garland (H.), Raghunathan (M.S.). - Fundamental domains for lattices in $\mathbb{R}$-rank 1 semisimple Lie groups, Ann. Math., t. 92, 1970, p. $279-326$.

[Gu] Guichardet (A.). - Cohomologie des groupes topologiques et des algèbres de Lie. - CEDIC-Fernand Nathan, Paris, 1980.

[KS] KorevaAR (N.), Schoen (R.). - Sobolev spaces and harmonic maps for metric space targets, Commun. Anal. Geom., t. 1, 1993, p. $561-659$.

[KT] KAniUth (E.), TAYLOR (K.F.). - Kazhdan constants and the dual space topology, Math. Ann., t. 293, 1992, p. 495-508.

[Lo] LotT (J.). - The zero in the spectrum conjecture. - Preprint, Univ. Michigan, 1996.

[Lu] Lubotsky (A.). - Discrete groups, expanding graphs and invariant measures, Progress in Math., t. 125, Birkhäuser, Basel, 1994.

TOME $126-1998-\mathrm{N}^{\circ} 1$ 
[LZ] Lubotsky (A.), Zimmer (R.). - Variants of Kazhdan's property for subgroups of semi-simple groups, Israel J. Math., t. 66, 1989, p. 289-299.

[M] Matsushima (Y.). - On the first Betti number of compact quotient spaces of higher dimensional symmetric spaces, Annals. of Math., t. 75, 1962, p. 312-330.

[Mo] MoK (N.). - Harmonic forms with values in locally constant Hilbert bundles, in Conference in honor of Jean-Pierre Kahane, Orsay 1993, J. Fourier Anal. Appl., special issue, 1995, p. 433-454.

[MSY] MoK (N.), SiU (Y.-T.), YEUnG (S.-K.). - Geometric superrigidity, Invent. Math., t. 113, 1993, p. 57-83.

[NR] Niblo (G.), Reeves (L.). - Groups acting on CAT(0) cube complexes, Geometry and Topology, t. 1, 1997, p. 1-7.

[P] PAnsu (P.). - Sous-groupes discrets des groupes de Lie : rigidité, arithméticité, Séminaire Bourbaki nº 778, Astérisque, t. 227, 1995, p. 69-105.

[R] Ronan (M.). - A construction of buildings with no rank 3 residues of spherical types, in Buildings and the geometry of diagrams, CIME conference at Como, 1984, Lect. Notes, t. 1181, Springer, Berlin, 1986, p. 242-248.

[RT] Ronan (M.), Tits (J.). - Building buildings, Math. Ann., t. 278, 1987, p. 291-302.

[S] Swiatkowski (J.).- - Polygonal complexes of nonpositive curvature : from local symmetry to global one. - Preprint, Univ. Wroclaw, 1994.

[T1] Tits (J.). - A local approach to buidings, in The geometric vein, Coxeter Festschrift, Springer, Berlin, 1981, p. 519-647.

[T2] Tits (J.). - Spheres of radius 2 in triangle buildings, in Finite geometries, buildings and related topics, Pingree Park Conference, 1988, Clarendon Press, Oxford, 1990, p. 17-28.

[WZ] Wang (McK.), ZILler (W.). - Symmetric spaces and strongly isotropy irreducible spaces, Math. Ann., t. 296, 1993, p. 285-326.

[Z] Zuk (A.). - La propriété (T) pour les groupes agissant sur les polyèdres, C. R. Acad. Sci. Paris, t. 323, 1996, p. 453-458. 